\title{
Through space and through bridge channels of charge transfer at p-n nano-junctions: a DFT study
}

\author{
Naveen Dandu, ${ }^{(b)}$ Sergei Tretiak $^{(a)}$, Svetlana Kilina ${ }^{(b)}$, Dmitri Kilin ${ }^{(b)}$ * \\ (a) Center for Integrated Nanotechnologies, Los Alamos National Laboratory, Los Alamos, 57069 NM \\ 87454 \\ (b) Department of Chemistry and Biochemistry, NDSU, Fargo, ND 58108
}

\begin{abstract}
Details of charge density distribution at p-n nano interface are analyzed with density functional theory techniques using model system of dimers of doped silicon quantum dots interacting through bond and through space. Spatial orbital distributions of transition densities between the ground and excited states suggest the character of essential electronic excitations, which have a Förster, bound, unbound, or charge transfer character. A redistribution of electronic density from n-impurities to p-impurities results in a ground state polarization and creates an offset of energies of the bands localized on p-doped quantum dot and the bands localized on n-doped quantum dot. Although impurities contribute very few orbitals to the total density, a ground state charge redistribution and polarization are both responsible for the presence of a large number of charge transfer excitations involving solely silicon orbitals.
\end{abstract}

KEYWORDS: arrays of quantum dots, p-n junction, depletion layer, drift current, photovoltaic effect, codoping, intrinsic silicon, optical properties, photodiode, transition density, charge transfer exciton, shallow impurity, TDDFT, exciton formation energy, bound exciton, 3D solids

(*) Corresponding Author, email: Dmitri.Kilin@ndsu.edu 


\section{I.INTRODUCTION}

Silicon based materials play a substantial role in microelectronics, photovoltaics, and light-emitting technologies. 1,2,3 Optoelectronic properties of these systems are modified by controlling type and concentration of dopants as well as by spatial confinement. ${ }^{4} \mathrm{~A}$ special interest is upholding on various implementations of interface between p-doped and n-doped silicon at nanostructured level. Formation of depletion layer, anisotropic conductivity, and control of interface polarization by optical excitation form a basis for the photovoltaic effect. The features of low-concentration doping in an infinite sample of bulk silicon have been tediously studied since $1940^{\mathrm{s}}$ for multiple industrial applications. Theoretical modeling of single pand $\mathrm{n}$ - shallow doping are offered through an analogy with a hydrogen atom. ${ }^{5}$ Properties of macroscopic junction between $\mathrm{p}$ - and $\mathrm{n}$ - doped regions are described in a phenomenological way by Schockley and Quisser. ${ }^{6}$ In recent decades, the working elements of electronic circuits and photovoltaic cells experience extreme miniaturization, following the Moore's law. ${ }^{7}$ Current microelectronic technology is based on elements ranging in the tens of nanometers. In an attempt at further miniaturization, the size of silicon-based working elements has approached the limit of a few nanometers. ${ }^{8}$ At such a limit, one expects two major effects affecting optoelectronic properties of silicon materials: size tuning of bandgap due to quantum confinement and atomistic nature of the doping. ${ }^{9,10}$ Specifically, for confined silicon materials, such as quantum dots, nanowires and thin films of characteristic length 1-10 nanometers, an impurity ion directly interacts with all available host neighbor atoms. In this limit, various effects of interfaces and surfaces of the nano-sized silicon are of substantial influence.

It was recently shown that silicon quantum dots placed in a dielectric matrix can form a material with a higher band gap than that of the bulk crystalline silicon and can, therefore, be applied as stable top solar cells for an all-silicon based tandem photovoltaic devices. ${ }^{11}$ Arrays of close packed quantum dot solids (QDs ) facilitate formation of exciton bands, increasing optical properties and promising more efficient photovoltaic devices. ${ }^{12}$ This promise, though, has been somewhat shadowed by theoretical predictions that resonant energy transfer between large $(\sim 2-4 \mathrm{~nm})$ Si QDs is possible only when they are almost in a contact, because of the relative efficiency of radiative relaxation. ${ }^{13,14}$ In a regime of close proximity, QDs may form a percolation network. ${ }^{15}$ One hypothesis is that the electronic structure of interacting QDs strongly depends on the presence or the absence of a covalent contact between them. This effect is of critical importance for understanding the formation of the p-n junction. The formation of a depletion layer and atomistic description of charge distribution at the interface between $\mathrm{p}$-doped and n-doped regions, and, more specifically, between p-doped and n-doped QDs, is a challenging question. Particularly, in the limit of small QDs and high concentration of doping, a substantial dependence of electronic state energy on the interfacial charge or exciton transfer is expected. In 1950s, Walter Kohn has introduced "Hydrogen-atom" model analogy for shallow dopants. ${ }^{5,16}$ These quantitative predictions of this model analogy are valid for both bulk materials and QDs. Atomistic modeling of planar p-n junction is computationally expensive due to larger size of the models. Most of available bulk calculations include only p- or only n- doped regions. A recently reported atomistic modeling of 
optoelectronic properties of doped silicon films ${ }^{17}$ provides analysis of orbitals contributed by dopants. For pure (un-doped) Si, orbitals are delocalized. For doped $\mathrm{Si}$, orbitals are partly localized on dopant and neighboring $\mathrm{Si}$ atoms illustrating analogy to hydrogen atom with Bohr radius of the order of 0.5 to $1.0 \mathrm{~nm}$. The band alignment is different in case of (i) interfaces in confined nanostructures, and in case of (ii) macroscopic interfaces in bulk and periodic slabs, as schematically illustrated in Figure S1. For model systems of size larger than the Si exciton size (i.e. its real-space delocalization), the situation would be similar to the regular macroscopic bulk p-n junctions, where free electrons are present away from the contact. At the very interface, however, the charge carriers are expected to undergo the re-arrangement similar to the one for nanostructures considered in this work. At larger distance from the interface, the n-states are expected to be occupied while the p-states will remain unoccupied. For modelling of a system with well-pronounced n-, p- and depletion layers, one needs to explicitly include more than one doping atom of each type. The inter- and intra- band transitions will spread over a larger number of energy states. ${ }^{17}$

Optoelectronic properties of small isolated silicon QDs have been studied using a broad arsenal of computational chemistry methods ranging from semi-empirical ${ }^{18,19,20,21,22,23}$ to Configuration-Interaction, ${ }^{24,25}$ multi-reference second-order perturbation theory MP2, ${ }^{26}$ Coupled Cluster, ${ }^{27}$ and diffusion quantum Monte Carlo $^{28,29,30}$ methods. The GW/BSE approach ${ }^{31,32,33}$ is a typical method to take into account a bound exciton state formation. Real-space pseudopotential methods ${ }^{34,35}$ and charge patching methods ${ }^{36}$ have been used to study larger Si and other semiconductor QDs. Among these approaches, methods based on Density Functional Theory (DFT) and time-dependent DFT (TDDFT) provide an optimal numerical tool for analyzing ground and excited state properties in a wide range of materials from molecules to semiconductor nanostructures, ${ }^{37,38}$ including surfaces, interfaces, irregular and distorted structures, as well as impurities. ${ }^{17}$ Practical applications of DFT and TDDFT have yet open questions on how computational results depend on methodological choices, i.e., various functionals, basis sets, and solvent models. Promising performance is reached with hybrid DFT models such as B3LYP, CAM-B3LYP, ${ }^{39} \mathrm{HSE}^{40,41}$, as well as DFT-D2/D3 methods of Grimme ${ }^{42}$ to account for dispersive inter-cluster interactions. Success of computational modeling of silicon nanomaterials is recorded for quantum dots, ${ }^{4,43}$ nanowires, ${ }^{44,45,46,47,48,49}$ and thin films ${ }^{50,51,52}$ for both intrinsic, single-type doping, and co-doping by $\mathrm{p}$ - and $\mathrm{n}$ - impurities at the same time. ${ }^{53,54,55}$

Additionally, much work has been done to study inter-dot interactions and the formation of collective states across QDs in an array in a view of technological relevance. The impact of the QD-QD separation on their opto-electronic properties has been recently considered. ${ }^{13,14,56,57,58,59}$ Energy transfer, charge transfer, and carrier multiplication (CM) effects, adopting a fully ab initio scheme within DFT, in both isolated and interacting H-passivated Si-QDs was reported. ${ }^{57}$ Computational modeling of interacting quantum dots shows noticeable modifications in four phenomena: (i) optical absorption, (ii) multiple exciton generation, (iii) charge transfer, and (iv) exciton formation. In particular we notice that: (i) optical absorption systematically increases across the broad spectrum as the H-terminated Si QDs approach each other; ${ }^{58}$ (ii) both colloidal silicon QDs ${ }^{60}$ 
and $\mathrm{Si}$ QDs embedded in $\mathrm{SiO}_{2}$ matrix ${ }^{61,62}$ demonstrate $\mathrm{CM}$ through multiple-exciton generation; (iii) at small separations ( $\sim 0.2 \mathrm{~nm}$ ) of silicon QDs in $\mathrm{SiO}_{2}$ matrix, the energy levels of the QDs are broadened to the minibands due to wave-function overlap, thus enabling electron transport, ${ }^{56}, 59$ (iv) provided that the QDs are sufficiently well separated, exciton hopping can appear within the framework of Förster resonance energy transfer, wherein the Coulomb interactions are approximated using either dipole-dipole ${ }^{63}$ or Dexter higherorder multipole interactions. ${ }^{64}$ Regarding the latter, Baer and Rabani ${ }^{65}$ investigated the relative contribution of various multipole interactions to resonance energy transfer, as well as to optical transitions near metal contact. ${ }^{66}$ Recently, Lin et al. ${ }^{14}$ have found that that small $(\sim 1 \mathrm{~nm})$ Si undoped QDs exhibit exciton transport efficiencies far exceeding that of their larger counterparts for the same center-to-center separation.

Overall, previous studies addressed in detail the electronic properties of isolated doped and undoped QDs, as well as interacting undoped QDs. A gap remains, however, in describing interactions of several doped quantum dots situated in close proximity. This work presents a systematic investigation of the mutual influence of the $\mathrm{p}$ - and $\mathrm{n}$ - doped quantum dots. This interaction is highlighted by comparing two types of atomic models with $\mathrm{p}$ - and $\mathrm{n}$ - regions being separated by vacuum or connected by a bridging silicon atom. Relative contributions of charge versus exciton transfer are elucidated by analyzing spatial distribution of transition density matrices corresponding to the essential electronic excitations. Our computational results suggest that the dominant transport mechanisms are influenced by the electronic structure of the p-n nano-interface. In arrays of molecularly connected quantum dots, the leading mechanism is an electron transfer; whereas in arrays of disconnected quantum dots, the leading mechanism is exciton transfer. These insights in charge vs exciton transport properties are expected to be helpful for technological applications in nano-electronics and photovoltaics.

The paper is organized as follows. Section II introduces details of computational methods and introduces the chemical composition of studied models. Results and discussion in Section III are organized is several subsections. Subsection III.a presents expected and computed trends in ground state electronic structure of QD dimers. Subsection III.b presents trends in absorption spectra and excited state profiles of QD dimers. Subsection III.c provides benchmarks for several options of QD modeling. Section IV concludes and summarizes main findings.

\section{II.COMPUTATIONAL METHODS}

The electronic structure of both ground and optically-excited states of a system can be efficiently explored in the DFT and TDDFT frameworks. Both rely on time-independent and time-dependent selfconsistent Kohn-Sham (KS) equations, respectively, and use concepts of total electronic density, total energy, and effective potential, including interactions of electrons with ions, and three electron iterations: Coulomb, correlation, and exchange. For consistent notation the equations of ground state DFT and TDDFT are mentioned in Supplemental Information. Computations are made with G09 software. ${ }^{67}$ 
Briefly, for the ground state, the solutions of KS equations provide set of orbitals $\left\{\varphi_{i}^{K S}\right\}$ and KS single-electron energies $\left\{\varepsilon_{i}\right\}$, which can be analyzed using the concept of density of states $D(\varepsilon)$ and density of occupied states $D^{\prime}(\varepsilon)$ as

$$
\begin{aligned}
& D(\varepsilon)=\sum_{i} \delta\left(\varepsilon-\varepsilon_{i}\right), \\
& D^{\prime}(\varepsilon)=\sum_{i} n_{i} \delta\left(\varepsilon-\varepsilon_{i}\right),
\end{aligned}
$$

where $\delta$ is a Dirac delta-function and $n_{i}$ is an occupation number. When adapting an approximation of uncorrelated single-particle states, the bandgap and band off-sets (see Figure 1) can be easily calculated. The band gap is defined differently for doped and undoped models. For undoped models we have the simplest case

$$
E_{g}=E_{c b}-E_{v b}=E_{L U}-E_{H O}
$$

Here we adopt terminology of molecular orbitals and conduction/ valence bands to denote individual levels and manifolds of orbitals, respectively. The terms "conduction band" and "valence band" have been originally introduced for condensed matter physics. However, there is a common convention and practice to use these terms in the QD research community. Motivations to use this notation are: (1) many orbitals contributed by host material, which have very similar energy, spatial distribution of charge density and contributions to spectra, and (2) convenience to highlight the differences between orbitals contributed by host material and orbitals contributed by doping. Thus all Si orbitals are referred to as CB and VB. We use the terminology of molecular (Kohn-Sham) orbitals, when referring to a specific orbital. However, as a common term for a manifold of occupied and unoccupied Si orbitals, we use "VB" "CB", respectively. Thus in equation (3a), $E_{L U}$ and $E_{H O}$ are energies of LUMO and HOMO orbitals, can be represented as energies of conduction band minimum $\left(E_{c b}, \mathrm{CB}\right)$ and valence band maximum $\left(E_{v b}, \mathrm{VB}\right)$, respectively.

For doped models the bandgap and difference between band edges are computed differently:

$$
E_{g}=E_{c b}-E_{v b}=E_{i}-E_{j}
$$

where $i(j)$ is the first orbital in the conduction (valence) band corresponding to a bulk material and is not introduced by dopant. For example, for $\mathrm{p}$-Si $i=L U+1, j=H O$; for the other models values of $i$ and $j$ are shown in Table 1. Shallow donor activation energy $E_{d}$ and shallow acceptor activation energy $E_{a}$ characterize a probability of thermal activation of a charge carrier from a localized impurity to the delocalized host band. When these values are comparable to thermal quantum $k T$, material exhibit an enhanced conductivity. Subsequently, $E_{d}$ and $E_{a}$ are defined as energy sub-gap between orbital contributed by a dopant and a relevant band edge: $\mathrm{CB}$ for a donor (here phosphorous) and VB for acceptor (here aluminum), defined as

$$
\begin{gathered}
E_{d}=E_{c b}-E_{d o p}=E_{L U-q}-E_{L U}, q=1 \\
E_{a}=E_{d o p}-E_{v b}=E_{H O}-E_{H O-q}, q=1
\end{gathered}
$$

More accurate approximation for correlated many-body electronic states is achieved in TDDFT approach via numerical solution of Casida equations. The latter provide a set of electronic transition energies $\left\{\hbar \Omega_{\alpha}\right\}$, with respective oscillator strength values for each transition $\left\{f_{\alpha}\right\}$ and transition density matrices $\left\{\Delta \rho^{\alpha}\right\}$, 
which are labeled by index of an excited state $\alpha$. Note that numerical procedure works for predefined number of excitations $\alpha<\alpha_{\max }$. Excited state properties can be analyzed using optical absorption

$$
a(\varepsilon)=\sum_{\alpha} f_{\alpha} \delta\left(\varepsilon-\hbar \Omega_{\alpha}\right)
$$

and density of excited states

$$
a^{\prime}(\varepsilon)=\sum_{\alpha} \delta\left(\varepsilon-\hbar \Omega_{\alpha}\right)
$$

An efficient analysis of excitation delocalization and comparison of charge transfer vs resonance energy transfer can be performed using the concept of transition density matrices ${ }^{37,38}$ providing a natural way to analyze spatial correlation of electron and hole forming an exciton. ${ }^{68,69}$ Earlier studies used this technique to analyze exciton localization in molecular dimers ${ }^{70,71}$ conjugated polymers $^{68,69}$ and carbon nanotubes. ${ }^{72,73}$ Specifically, in the atomic basis, the elements of transition density matrix can be defined as a matrix element of an electron annihilation on orbital $A^{\prime}$ and an electron creation on orbital $A$ upon photoexcitation from ground $|g\rangle$ to excited $|\alpha\rangle$ states

$$
\Delta \rho_{A, A^{\prime}}^{\alpha}=\left\langle\alpha\left|\hat{c}_{A}^{+} \hat{c}_{A^{\prime}}\right| g\right\rangle
$$

The discrete two-dimensional plots $\left|\Delta \rho_{A, A}^{\alpha}\right|$ approximately represent the mutual location of electrons (X-axis) and holes (Y-axis). Plots with maximal distribution of diagonal values indicate strongly bound excitons, offdiagonal areas correspond to electrons and holes delocalized across the system. Charge transfer excitations appear in the off-diagonal segment.

For better visualization of transition density maps, atomistic structures for numerical investigation of the formation of the nano p-n junction are presented in Figure 1. Typically, the experimentally synthesized QD sizes range from 2 to $15 \mathrm{~nm} .{ }^{74}$ In this work one models a small sized QDs to ensure the faster simulations, since simulation of dimers of larger sized QDs would be numerically intractable. While the size of computationally modelled QD's are typically smaller than that of the synthesized QDs, the qualitative trends would apply to larger size QDs as well. This paper presents analysis of charge transfer between two QDs whose electronic structure mostly depend on doping pattern. Energies of levels introduced by dopants are expected to repeat the same pattern for broad range of QD sizes, while the absolute value of the gap of QDs depends on its quantum confinement in a systematic and predictable way: the larger the QDs diameter, the smaller is the band gap toward saturation to the bulk limit. The trends of size dependence of Si QD bandgaps are illustrated in Figure S2.

The selected atomistic models of Si QDs were cut from a bulk crystalline Si. Electronic properties of QDs share some features characteristic for both solids and molecules. In QDs, similar to molecules, the momentum of electrons is not a good quantum number, however, similar to solids, the orbital energies are densely spaced. Therefore, the DOS for QD is very similar to the DOS of the bulk semiconductor of the same chemical composition. The quasi-continuous collections of dense orbitals can then be referred to as "bands", in what follows. 
Two sets of models are based on pair of nearly spherical quantum dots of the 35-atom size, terminated with hydrogens. In both the geometries, QDs are interfacing each other in the $<112>$ direction. The disconnected dimer consists of two silicon quantum dots with 70 atoms in total. In the bridged dimer, two quantum dots share one common silicon atom, with the total number of atoms being 69. In addition, we replace the central atom in each quantum dot in both structures by either one of the following elements: $\mathcal{A}, \mathcal{B}$ $=\mathrm{Si}, \mathrm{Al}, \mathrm{P}$. The replacement of silicon by aluminum and phosphorous implements $\mathrm{p}$ - and $\mathrm{n}$ - doping, respectively, since dopant atoms have ionic charge varied by plus or minus one. Chemical compositions of both models read

$$
\begin{aligned}
& \mathcal{A}_{1} \mathrm{Si}_{34} \mathrm{H}_{36}+\mathcal{B}_{1} \mathrm{Si}_{34} \mathrm{H}_{36} \\
& \mathrm{H}_{34} \mathcal{A}_{1} \mathrm{Si}_{34}-\mathrm{Si}_{-}-\mathrm{Si}_{34} \mathcal{B}_{1} \mathrm{H}_{34}
\end{aligned}
$$

Altogether, in addition to 3 monomers, we have considered 8 distinct dimer structures, namely $2 \mathrm{x}$ $\mathrm{Si}_{35} \mathrm{H}_{36}$ (i-Si), 2 x Al $\mathrm{Ai}_{34} \mathrm{H}_{36}$ (p-Si), $2 \times \mathrm{P}_{1} \mathrm{Si}_{34} \mathrm{H}_{36}$ (n-Si) and $\mathrm{Al}_{1} \mathrm{Si}_{34} \mathrm{H}_{36}+\mathrm{P}_{1} \mathrm{Si}_{34} \mathrm{H}_{36}$ (pn-junct.) for both bridged and disconnected configurations.

In each atomic model, we further specifically label each ion according to its separation from the central atom along Z-direction, i.e., the ion positions $\vec{R}_{I}=\left(X_{I}, Y_{I}, Z_{I}\right)$ grow with $I^{\text {th }}$ index, $Z_{I-1}<Z_{I}<Z_{I+1}$. Such specific ordering of ions is directly related to the spatial distribution of the electronic density. An electron

occupying an atomic orbital $\varphi_{i}\left(\vec{R}_{I}-\vec{r}\right)$ is localized in the vicinity of space region $\vec{R}_{I} \pm d \vec{r}$. Therefore, an atomic orbital index indicates the position of an electron in space. Electronic structures of all dimers were computed with B3LYP functional and LANL2DZ basis set. The validity of this model chemistry for silicon nanostructures is introduced in equations (7) and (8) and the details are discussed at the end of the results section.

\section{III.RESULTS AND DISCUSSIONS}

III.a. Ground state electronic structure of $Q D$ dimers

Qualitative schematic diagram of the band structure of the nano-scale p-n junction is shown in Figure 2. Most important features of electronic structure are energy offsets between band edges and shallow impurity levels. Computational modeling can quantify values of such offsets and their mutual alignments. Here, we are interested in the values of band-gap, doping activation energy, and offsets between silicon bands. The interaction of $\mathrm{n}$ - and p-doped QDs facilitates electron transfer from n-doped to p-doped QD, in the ground state, compared to the ground state of non-interacting QDs. Upon photoexcitation of such p-n nano-interface, the electron undergoes backward transfer from p-doped to n-doped QD. This section contains analysis of ground state only. Notably, upon electron transfer through bond contact between $p$-doped and $n$-doped regions the bandgap is expected to decrease. Upon bringing $p$-doped and $n$-doped quantum dots together as shown in Figure 2(e), we observe the following effect: (i) Drift of a charge density from $n$-impurity to $p$-impurity supported by the values of the total Mulliken charge extracted from Gaussian output files of Al- and P-doped 
QDs, (ii) systematic shift of energies for all bands, specifically to the lower energies at $n$-doped side and to the higher energies at $p$-doped side. The model exposes a spatial region located in space between $\mathrm{Al}$ and $\mathrm{P}$ atoms, where charge density experiences substantial change due to co-doping. This area has some features similar to those of depletion layer in planar semiconductor interface. ${ }^{75}$ The term depletion layer is typically used to label the layer separating hole and electron doped materials in the planar interface as shown in Figure S1. There, the redistribution of charge carriers at the interface can be rationalized from several aspects: (a) Microscopically, electrons subsequently occupy orbitals from low to high energy, to follow Fermi distribution, (b) Electrostatically, the difference in Fermi energy of $\mathrm{p}$ - and $\mathrm{n}$ - regions leads to a respective difference in potentials and the interfacial electric field. The latter drives charge carriers into opposite directions, (c) Mulliken charge analysis reported in Figure S3 provides signatures of such distinct charge redistribution and (d) since our models contain an exaggerated high concentration of dopants, the size of this layer is very narrow.

Density of states for each structure are calculated according to Eqs. (1)-(2), are shown in Figure 3 and are summarized in Table 1 and Figure 4. Analysis of data in Figure 4 is provided for both disconnected and bridged models. Analysis of trends in bandgaps and activaltion energies is performed in comparison with literature values. Specifically, measured values of $E_{d}$ and $E_{a}$ are available from IR spectroscopy. ${ }^{76}$ Theoretical predictions for $E_{d}$ and $E_{a}$ in bulk Si are available from hydrogenic model of shallow impurities, introduced by W. Kohn. ${ }^{5}$ Computational predictions of $E_{d}$ and $E_{a}$ in doped quantum dots are available from works. ${ }^{77,78}$

We start our discussion with comparison of electronic properties of all cases studied by inspecting the relationship between the values of $E_{g}, E_{a}, E_{d}$, and $E_{c b-v b}$. First of all, a systematic trend is observed for $E_{g}=E_{c b-v b}$. In all models, connected and disconnected, CB-VB bandgap value follows the sequence

$$
E_{c b-v b}(i-S i)>E_{c b-v b}(\square-S i)>E_{c b-v b}(n-S i)>E_{c b-v b}(p n-j u n c t .) .
$$

Interestingly, for structures with equivalent composition,

$$
E_{c b-v b}^{(\text {disconnected })}>E_{c b-v b}^{(\text {bridged })} .
$$

The trend in Eq. (10) holds for all models: $i-S i, n-S i, p-S i$ except the $p-n$ junction. For $p-n$ junction we found that

$$
E_{c b-v b}^{(\text {disconnected })}(p n-j u n c t .)<E_{c b-v b}^{(\text {bridged })}(p n-j u n c t .)
$$

We hypothesize that in the bridged dimer, charge density is more dynamically delocalized, thus effectively reducing the polarization screening: The effective dielectric constant $\epsilon_{\text {eff }}$ is higher in the bridged dimer model. Therefore, the Coloumb component $\left(\epsilon_{0} \epsilon_{e f f}\right)^{-1}\left|r_{p}-r_{n}\right|^{-1}$ is smaller in the bridged dimer compared to the disconnected counterpart. Furthermore, the carrier activation energies are systematically smaller in the disconnected dimers 


$$
\begin{aligned}
E_{a}^{(\text {disconnected })} & <E_{a}^{(\text {bridged })} \\
E_{d}^{(\text {disconnected })} & <E_{d}^{(\text {bridged })}
\end{aligned}
$$

since interaction through the bridge (through bond) provides stronger splitting.

The respective spatial distributions of selected Kohn-Sham orbitals are displayed in Figure 5. In the disconnected model, all but one (HO) highest occupied orbitals are localized on the p-doped quantum dot with $\mathrm{Al}$ ion in the center, while all but one (LU) lowest unoccupied orbitals are localized on the n-doped quantum dot with $\mathrm{P}$ ion in the center. In the bridged model, all highest occupied orbitals are localized on the $p$-doped quantum dot with $\mathrm{Al}$ ion in the center, while all lowest unoccupied orbitals are localized on the $n$-doped quantum dot with $\mathrm{P}$ ion in the center. In such configurations, the lowest energy excitations are prompted to have charge transfer character. It has been reported that co-doping of Si QDs contributes band edge states (HOMO and LUMO), spatially localized in the vicinity of the dopants. ${ }^{79,80}$ This feature lowers the energy gap compared to that of pure Si QDs. In some sense, DOS of co-doped Si QDs looks similar to the respective DOS of pristine Si QDs albeit with lower energy gap.

Disconnected dimers: In the $p$-doped quantum dot, the Al-dopant contributes an empty orbital, $\mathrm{LU}_{\mathrm{Al}-\text {-doped, }}$ near the top of the valence band, see a vacant orbital in the DOS, Figure 3(c) at about $-5.7 \mathrm{eV}$. In contrast, in the $n$-doped quantum dot, the P-dopant contributes an occupied orbital, $\mathrm{HO}_{\text {P-doped, }}$, near the bottom of the conduction band (see filled orbital in DOS, Figure 3 (d) at about $-2.7 \mathrm{eV}$ ). The Fermi energy of two quantum dots experiences equilibration: All orbitals of Al-doped quantum dot consistently shift higher up in energy. All orbitals of the P-doped quantum dot consistently shift down in energy. As a result, for the model containing two alternatively doped quantum dots, the lowest unoccupied orbital remains localized on the Al-doped quantum dot, while the highest unoccupied orbital remains localized on the P-doped quantum dot. The $p$ - $n$ junction structures are polarized. As can be seen from Figure 3(h), additional states appear at the edges of conduction and valence bands due to $n$ - and p-doped impurities. The small size of the model, the short distance between dopants, and high level of orbital hybridization promote ground state of co-doped model into intrinsic configurations. This effect was computationally investigated in reference. ${ }^{53}$ The charge density distribution between quantum dots is manifested by a significant total ground state dipole moments of the dimers representing $p$-n junction, which equal $\sim 65$ (36) Debye for disconnected (bridged) structures. Mulliken charge analysis for co-doped QD dimers, shown in Figure $\mathbf{S 3}$ demonstrates the charge imbalance at the doping ions. Aluminum has charge $+0.81(+0.79)$ in disconnected (bridged) dimers. Phosphorus has charge of $-0.56(-0.58)$ in disconnected (bridged) dimers. The charge on $\mathrm{Si}$ ions is noticeably smaller. Similar values of charge imbalance but different distances between $\mathrm{Al}$ and $\mathrm{P}$ ions result is different values of ground state dipole. 
Bridged dimers: Here, two alternatively doped quantum dots have a single common silicon atom, pipelining the charge density between dots. In this model, the equilibration of Fermi energies between individual quantum dots is attained by a transfer of an electron from the P-ion orbital in the $n$-doped quantum dot to the Al-ion orbital in the p-doped quantum dot, as shown in Figure 5: the highest occupied orbital is localized on Al-doped quantum dot and lowest unoccupied orbital is localized on P-doped quantum dot. This is different from the situation with disconnected quantum dots, which suggests that bridging $p-S i$ and $n-S i$ promotes an electron transfer from $n-S i$ to $p-S i$ symbolically represented as a reaction

$$
\begin{aligned}
& A l^{(0)} P^{(0)} \underset{\text { bridging }}{\longrightarrow} A l^{(\delta-)} P^{(\delta+)} \\
& A l^{(0)}+e^{-} \underset{\text { bridging }}{\longrightarrow} A l^{(-)} \\
& P^{(0)} \underset{\text { bridging }}{\longrightarrow} P^{(+)}+e^{-}
\end{aligned}
$$

Silicon atoms in the vicinity of n-doping (p-doping) have more positive (negative) charge compared to the average value. The charge on silicon atoms between the dopants is perturbed at most. The charge of Si atoms apart from the space between $\mathrm{P}$ and $\mathrm{Al}$ experiences smaller modification. The charges on atoms on the line connecting dopants, indicated by circles, follow the pattern:

$$
\mathrm{Si}^{+0.21}-\mathrm{Si}^{-0.03}-\mathrm{P}^{-0.54}-\mathrm{Si}^{-0.04}-\mathrm{Si}^{-0.15}-\mathrm{Si}^{-0.31}-\mathrm{Al}^{+0.79}-\mathrm{Si}^{-0.32}-\mathrm{Si}^{+0.12} .
$$

This observed density redistribution is supported by Mulliken charge analysis in Figure 4(c). P and Al experience the largest change of charge.

This redistribution of charge density also establishes an interfacial dipole from P-doped to the Al-doped quantum dot, as elucidated by the projection of the total dipole moment on to z-direction for the disconnected and bridged models of two alternatively doped quantum dots in the ground state. This equals $\mu_{z}^{\text {disconnected }=}$

65 Debye, and $\mu_{z}^{\text {bridged }}=36$ Debye, while all other dipole moment components for these two and for other models are much smaller, as shown in Supplemental Table S1.

The most important features of the electronic structure of considered models are dictated by a few states contributed by doping. Mutual energies of such band edge localized states have vanishing dependence on the size of QD. Note that for small-size co-doped systems at high concentration of $\mathrm{n}$ - and p- dopants, the populations of levels contributed by co-dopants swap according to the Fermi distribution, see Figure S4(d).

\section{III.b Spectra and excited state profiles of $Q D$ dimers}

Optical absorption spectra calculated using Eq. (4) are shown in Figure 6 for all dimers. TDDFT calculations were performed for $\alpha_{\max }=200$ excited states for each model, which allows exploring different ranges of transition energies, up to $5.5 \mathrm{eV}$ for un-doped, up to $5 \mathrm{eV}$ for $\mathrm{p}$-doped, up to $4.5 \mathrm{ev}$ for n-doped, and up to $3.5 \mathrm{eV} / 4.5 \mathrm{eV}$ for disconnected/bridged pn-doped models. Notably, for the analogous atomic composition, disconnected and bridged models do have a very similar density of excited states, computed with Eq. 5 . The 
analysis of density of excited states and absorption spectra provides several trends for peak energy and brightness.

(i-Si). For undoped models presented in Figure 6(a)-(b), the energies of the first transitions are $5.1 \mathrm{eV}$ and $4.8 \mathrm{eV}$, for disconnected and bridged dimers, respectively. This is consistently lower by $\Delta \approx 0.5-0.6 \mathrm{eV}$ than the ground state bandgaps of $5.7 \mathrm{eV}$ and $5.3 \mathrm{eV}$ for the same models. We observe a uniform blue-shift of transition energies/bandgaps by $\Delta$ consistent with earlier trend computed for CdSe quantum dots ${ }^{81,82}$ and doped Si quantum dots. ${ }^{54}$ Notably, $\Delta$ shift does not necessarily measure the exciton binding energy; this shift results from an interplay of many factors, including orbital relaxation, exchange, Coulomb interactions, taking care of self-interaction error, etc. ${ }^{83}$

(p-Si) and (n-Si), disconnected. Spectra of the disconnected models doped by the same impurity are presented in Figure 6(c),(e). For all of them, the first excitation transition energies are very close to zero. A common spectral feature of all these models is that there is a substantial density of excited states in the entire range of energies below the optical bandgap. A very few of these excited states are optically allowed (bright). The energies of bright peaks are spaced homogeneously throughout the whole bandgap range with about six bright peaks total. The energies of the bright peaks in the disconnected models $2 \times \mathrm{Al}_{1} \mathrm{Si}_{34} \mathrm{H}_{36}$ and $2 \mathrm{x}$ $\mathrm{P}_{1} \mathrm{Si}_{34} \mathrm{H}_{36}$, approximately match the computed energies of the bright peaks of the single isolated QDs $\mathrm{Al}_{1} \mathrm{Si}_{34} \mathrm{H}_{36}$ and $\mathrm{P}_{1} \mathrm{Si}_{34} \mathrm{H}_{36}$ dots as reported in the literature. ${ }^{43}$ The comparison is presented in Table 2. We hypothesize that these bright peaks correspond to transitions between orbitals contributed by dopants, bringing extra states to the system.

(p-Si) and (n-Si), bridged. Spectra of the bridged models doped by same type of impurity are shown in Figure. 6(d),(f). The connection through a bridge does not notably affect the density of excited states, however, we observe a substantial increase of the oscillator strength of the excitations with higher energy. We hypothesize that there is a constructive addition of transition dipole moments upon bridging or a contribution of the delocalized Si-Si optical transitions, which appear at the lower energy in the bridged models.

(p-n-Si junctions) Spectra of the disconnected and bridged dimers are shown in Figure 6(g)-(h). Their specific features are highlighted in Table 3 and Figure 7. Transition energies are described with the same notation style as for ground state orbitals values of $E_{g}, E_{a}, E_{d}, E_{c b-v b}$. Based on a comparison of the single electron states and the density of excited states, we interpret the four distinct peaks in Figure 6(g)-(h) as the following transitions are in the ascending order of their energies. $\left(E_{g}\right)$ is a transition between p- and nstates, providing the lowest excitation at the bandgap and likely associated with long-range charge transfer. $\left(E_{g}+E_{d}\right)$ is an excitation where the hole is localized on one QD and an electron promoted higher up into the $\mathrm{CB}$ becomes delocalized across the dimer. $\left(E_{g}+E_{a}\right)$ is an opposite case where the electron is localized, while a hole down in the VB becomes delocalized. Finally, $\left(E_{c b}-E_{v b}\right)$ is an excitation where both an electron and a 
hole are spatially delocalized. Interestingly, the values of these four specific transition energies are consistently blueshifted in the bridged model by about $1 \mathrm{eV}$.

$$
\begin{aligned}
E_{g}^{(\text {disconnected })}<E_{g}^{(\text {bridged })} & \\
E_{g}^{(\text {disconnected })}+E_{d}^{(\text {disconnected })} & <E_{g}^{(\text {bridged })}+E_{d}^{(\text {bridged })} \\
E_{g}^{(\text {disconnected })}+ & E_{a}^{(\text {disconnected })}<E_{g}^{(\text {bridged })}+E_{a}^{(\text {bridged })} \\
E_{c b-v b}^{(\text {disconnected })} & <E_{c b-v b}^{(\text {bridged })}
\end{aligned}
$$

This trend is attributed to the screening effects as discussed above.

In the bridged dimer model, the $\left(E_{c b}-E_{v b}\right)$ transition is noticeably brighter than in the disconnected dimer model. The intensity of absorption at a given frequency changes by about an order of magnitude: a bridged dimer model provides brighter excited states with respect to the disconnected dimer model of the same composition. We discuss two possible explanations of this effect: (i) Our simulations of both bridged and disconnected dimers used $\alpha_{\max }=200$ excitations. In the disconnected case, we observe the systematic shift of orbitals, up for $\mathrm{p}-\mathrm{QD}$ and down for $\mathrm{n}-\mathrm{QD}$, which is larger by about $1 \mathrm{eV}$ compared to the bridged system. All $\alpha_{\max }=200$ lowest excitations in the disconnected model have partial or full charge transfer character, from $p$ - $V B^{\prime}$ to $n-C B$ ". This offset of bands is smaller in the other case. Therefore, some high energy excitations among $\alpha_{\max }=200$ states have a neutral character, from $p-V B^{\prime}$ to $p-C B^{\prime}$ and from $n-V B$ " to $n-C B$ ". Such excitations are likely optically bright. (ii) Charge transfer excitations between p-QD and n-QD have different oscillator strengths in the disconnected and bridged models: $p V B^{\prime} \rightarrow n C B^{\prime \prime}$. A high intensity of such an excitation is prohibited in the disconnected model, since orbitals localized on p-QD and on n-QD are separated by an empty space region and have negligible overlap. In the bridged model, the spatial overlap of $p V B^{\prime}$ and $n C B^{\prime \prime}$ orbitals increases and enhances probability to create such charge transfer state by optical excitation.

The transition density spatial profiles for the lowest excitations are presented in Figure 8, according to Eq. (6). Additional transition density images are provided in Figure S5. The following features are observed: (i) Disconnected undoped dimer $(i-S i)$ shows the same profiles of transition density in segments corresponding to neutral excitation at each monomer. It can be interpreted as a superposition of degenerate monomer excitations, a delocalized excitation, or a hybridization of a monomer excitation achieved through space via Förster coupling. (ii) Bridged undoped dimer ( $i-\mathrm{Si}$ ) shows substantial transition density on molecular junction atom. (iii) Disconnected and bridged dimers doped by the same type of impurity exhibit degenerate charge transfer excitations. Interestingly, the degeneracy is lower for the ( $n$-Si) bridged model. (iv) Both co-doped ( $p$ $n \mathrm{Si}$ ) disconnected and bridged dimers feature charge transfer excitons without degeneracy.

Most intriguing results were obtained for $\mathrm{p}$-n doped dimers. We have found a numerical support for (a) a charge density redistribution in donor-acceptor dimer in its ground state and (b) a low energy charge transfer excitation induced by photon.

III.c Benchmarks of methods for QD modeling 
Finally, to benchmark various functional models, $\mathrm{Si}_{29}\left(\mathrm{CH}_{3}\right)_{36}$ QD was optimized using five different functionals, namely, B3LYP, PBEPBE, PBE1PBE, CAM-B3LYP and LC-wPBE featuring different amount of orbital exchange. For all calculations, 6-31 $\mathrm{g}^{*}$ basis set was used. The obtained band gap values are reported in Table 4. The B3LYP calculated band gap showed the best agreement with the experimental value. A comparison of densities of states and absorption spectra computed with different DFT models demonstrate that, while the values of gap energies are different, the main qualitative features of bands are very similar in all the cases as shown in Figures 9 and 10, respectively. To obtain the absorption spectra as shown in Figure 10, we have performed TDDFT to compute 50 excited states and plotted them using a band width of $0.05 \mathrm{eV}$.

\section{IV.CONCLUSIONS}

The interaction of semiconductor quantum dots leads to distinct signatures in their optical and electronic properties. A number of interesting effects are observed by comparing through-bond (bridged) and through-space (disconnected) types of interaction of silicon quantum dots in our dimer models. The electronic structure and optical properties of both bridged and disconnected interacting QDs dimers show strong dependence on the doping pattern. Dimers functionalized with impurities, single-type doped or co-doped, exhibit similar qualitative properties. In contrast, the un-doped dimers manifest noticeable qualitative differences. Specifically, the bridged un-doped dimer shows an order of magnitude brighter lowest exciton transition. The spatial profile of the lowest exciton transition density in bridged dimer models shows signatures of electron-to-hole interaction facilitated by bridging atom. The disconnected and undoped dimers shows lowest exciton character interpreted as Förster energy transfer. For both doped connected and disconnected dimers, the lowest excitation forms a charge-transfer degenerate exciton band, which appears due to doping with the same impurity type.

The most important case of interface between $\mathrm{p}$-doped and $\mathrm{n}$-doped regions, represented by aluminum and phosphorous, respectively, shows clearly defined direction of polarization of the interface. In the lowest excited state, an electron is localized on the phosphorous side (n-doping) and a hole is localized on the aluminum (p-doping) side of the interface. This result is consistent for both bridged and disconnected models. The transition between ground state to the lowest excitation at the $\mathrm{p}-\mathrm{n}$ interface is accompanied by a change of the polarization at the interface. According to our calculations, the ground state is more polar than the lowest excited state. A cyclic excitation and de-excitation of the p-n-junction leads to a change in the polarization value for the entire structure. In a hypothetical presence of the bias contacts, these processes would illustrate the photovoltaic effect at the p-n-interface.

In addition to excitonic properties of the $\mathrm{p}-\mathrm{n}$ interface, reported computations provide useful quantitative measures of carrier activation energies. The bridging or separation of the $\mathrm{p}$ - and $\mathrm{n}$ - regions brings noticeable variations to the carrier activation energies: hole activation energy for p-doped Si models and electron activation energy for n-doped models. For each type of doping, the carrier activation energy is larger in the bridged models. Obtained structure-property relations provide insights to our understanding of basic 
electronic interaction mechanisms in super-solids: macroscopic arrays of ordered quantum dots fabricated for broad range of opto-electronic applications including photovoltaic and lighting.

\section{V.SUPPLEMENTAL INFORMATION}

Technical details of the methodology and validation of used approach are available in the Supplemental information. Additional data are provided for ground state static dipoles of considered models along with transitional density maps for several excitations for each model studied in the paper: un-doped and co-doped with $\mathrm{Al}$ and $\mathrm{P}$ atoms in Si-quantum dots.

\section{ACKNOWLEDGEMENTS}

D.K. together with S.K. thank NSF grant CHE-1413614 for financial support of methods development. We acknowledge support of Center for Integrated Nanotechnology (CINT) and Center for Nonlinear Studies (CNLS) at Los Alamos National Laboratory (LANL). LANL is operated by Los Alamos National Security, LLC, for the National Nuclear Security Administration of the U.S. Department of Energy under contract DEAC52-06NA25396. S.K. acknowledges financial support of the U.S. Department of Energy (DOE) Early Career Research grant DE-SC008446 for the work on nanostructures and the Alfred P. Sloan Research Award BR2014-073 for the work on method justification. D. S. K. thanks Mariah Hoffman for discussions and editorial suggestions.

\section{TABLE CAPTIONS}

Table 1. Summary of ground state electronic structure calculations for eight atomic models of doped quantum 
dots, computed with B3LYP functional and LANL2DZ basis. The computation of bandgap needs a comment. For undoped models, $E_{g}=E_{c b}-E_{v b}=E_{L U}-E_{H O}$. For doped models the bandgap and difference between band edges are computed differently: $E_{g}=E_{L U}-E_{H O}$ but $E_{c b}-E_{v b}=E_{i}-E_{j}$, where $i$ is the first orbital not contributed by doping in the conduction band, $j$ is the first orbital not contributed by doping in the valence band. Specifically, for $\mathrm{p}-\mathrm{Si}$ $i=L U+1, j=H O$; for other models values of $i$ and $j$ are shown in table. Donor activation energy $E_{d}$ and acceptor activation energy $E_{a}$ are defined as energy sub-gap between orbital contributed by dopant and relevant band edge: $\mathrm{CB}$ for donor, which is phosphorous and VB for acceptor, which is aluminum. Measured values of $E_{d}$ and $E_{a}$ are available from IR spectroscopy. ${ }^{76}$ Theoretical predictions for $E_{d}$ and $E_{a}$ in bulk Si are available from hydrogenic model of shallow impurities, introduced by W. Kohn. ${ }^{5}$ Computational predictions of $E_{d}$ and $E_{a}$ in doped quantum dots are available from works. ${ }^{77,78}$ For suspicious cases, alternate values are computed as indicated in footnotes and nearly match labels in Figure 2. Specific pairs of orbitals are indicated with (*) symbols to avoid ambiguity in the notation as follows: ${ }^{*} \mathrm{E}(\mathrm{LU}+1)-\mathrm{E}(\mathrm{HO}-1),{ }^{* *} \mathrm{E}(\mathrm{LU}+1)-\mathrm{E}(\mathrm{HO}-3),{ }^{* * *} \mathrm{E}(\mathrm{LU}+1)-$ $\mathrm{E}(\mathrm{HO}),{ }^{* * * *} \mathrm{E}(\mathrm{LU})-\mathrm{E}(\mathrm{HO}-1),{ }^{* * * * *} \mathrm{E}(\mathrm{HO})-\mathrm{E}(\mathrm{HO}-3)$.

Table 2. Absorption below bandgap in p- and n- doped, disconnected models. Comparison of transition energies of bright absorption peaks, in eV. Literature value are taken from reference Mavros et al., ${ }^{43}$ Fig. 3(b)* and Fig. 3(f)**

Table 3. Energies of peaks in the density of excited states of the disconnected and bridged p-n junctions, presented in Fig. 6(g), 6(h). X-axis show hypothetic labels, which might correspond to these transitions.

Table 4: Band gap in eV of Si29 QD with different functionals.

\section{FIGURE CAPTIONS:}

Figure 1. Optimized geometry of (a) disconnected and (b) bridged models. All models were carved from bulk $\mathrm{Si}$ crystalline structure to the pairs of minimalistic Si-QD models of composition $\mathrm{Si}_{35} \mathrm{H}_{36}$. All models are oriented along $\mathrm{Z}$-axis, so that second QD is a translation along $Z$. It is critical to note that the positions 
$\vec{R}_{I}=\left(X_{I}, Y_{I}, Z_{I}\right)$ of each ion in the model are ordered $Z_{I-1}<Z_{I}<Z_{I+1}$ so that values of Z-projection of position of each ion grow with index of $I^{\text {th }}$ ion in the model. Resulting composition for the disconnected model reads $2 \times \mathrm{Si}_{35} \mathrm{H}_{36}$. For the bridged model, two QDs are brought to the closest vicinity, one $\mathrm{Si}$ atom, closest to the neighboring QD is removed and its place is aligned with the nearest $\mathrm{Si}$ atom in the second QD with two hydrogens are also removed to keep the surface termination pattern. Resulting atomic composition of the bridged model reads $\mathrm{H}_{34} \mathrm{Si}_{34}-\mathrm{Si}_{-} \mathrm{Si}_{34} \mathrm{H}_{34}$. Central $\mathrm{Si}$ atom in each of Si-quantum dots was either kept intact or replaced with $\mathrm{Al}$ or $\mathrm{P}$ ions. Interestingly, upon the geometry optimization the disconnected QDs slightly rotate in the XY plane with respect to each other.

Figure 2. Schematic representation of the formation of nano p-n interface. (a) undoped model, (b) p-doped model obtained replacing one $\mathrm{Si}$ atom with one $\mathrm{Al}$ atom, (c) n-doped model obtained by replacing one $\mathrm{Si}$ atom with one P-atom, (d) nano-sized p-n junction, and (e) schematic diagram of charge transfer. Here, yellow/cyan boxes represent conduction and valence bands, respectively, continuous lines represent filled orbitals, and dashed lines represent unoccupied orbitals. Arrows represent key features of electronic structure, $E_{g}, E_{a}, E_{b}$, $E_{c b-v b}$, according to Eqs. (3). Note that each dopant contributes more than one additional state. Only the most important shallow states in the bandgap range are shown for simplification. Excitonic effects at $p-n$ junction contribute to the substantial reduction of the bandgap. At the $p$-n junction, energies of all bands at $p$-doped side, CB', VB' and all bands at n-doped side, CB”, VB" experience different systematic shifts in energies. Specifically, energies of all bands at p-doped side increase, while energies of all bands at $\mathrm{n}$-doped side decrease. This is illustrated by relative shift of yellow and cyan boxes in panel (d). Note that upon formation of $\mathrm{p}$ - $\mathrm{n}$ junction there is a drift current from $\mathrm{n}$-doped side to $\mathrm{p}$-doped side This drift forms a depletion layer and interfacial dipole. The polarization, interface field, and impurity band offset achieve equilibrium values. Optically active transitions involve pairs of orbitals at either p-side or n-side of the interface as symbolized by blue dashed arrows.

Figure 3. Density of states of the ground state electronic structure calculations for eight atomic models of pairs of doped quantum dots, computed with B3LYP functional and LANL2DZ basis. Filled area under the curve indicates occupied orbitals. Panels (a), (c),(e),(g) represent pairs of disconnected QDs. Panels (b), (d),(f),(h) represent bridged QDs chemically bonded to each other through bridging silicon atom. Panels (a)-(b) represent un-doped QDs and labeled as intrinsic silicon (i-Si). Panels (c)-(d) represent aluminum doped QDs and are labeled as p-Si. Panels (e)-(f) represent phosphorus doped QDs and are labeled as n-Si. Panels (g)-(h) represent results for models where one QD in the pair is doped by aluminum and second one by phosphorous, therefore representing p-n junction. Data in panels $(\mathrm{g})$ and $(\mathrm{h})$ is one of the important result of the paper. Bandgap energy $E_{g}$ along with donor activation energy $E_{d}$ and acceptor activation energy $E_{a}$ are labeled as energy spacing between feature peaks in DOS. 
Figure 4. Comparison of the $E_{c b-v b}$ bandgap values for disconnected (+) and bridged (x) models. Data correspond to Table 1, column IV. In all models except p-n junction, the bridged models have a smaller value of the $E_{c b-v b}$ bandgap.

Figure 5. Kohn-Sham orbitals for atomistic models of co-doped quantum dots, which represent disconnected (left) and bridged (right) p-n junction. Colored spheres represent atoms of Si (large cyan spheres), H (small cyan spheres), $\mathrm{Al}$ (pink), $\mathrm{P}$ (yellow). Red and blue clouds represent iso-surfaces $\left|\operatorname{Re}\left(\varphi_{\mathrm{i}}\right)\right|=0.02$ of real part of spatial distribution of Kohn-Sham orbitals. Positions of models are slightly rotated for better visualization and comparison of orbitals. For each model, four highest occupied, HO-3 ... HO and four lowest unoccupied, LU ... LU+3 orbitals are shown. In the disconnected model, all but one (HO) highest occupied orbitals are localized on the p-doped quantum dot with $\mathrm{Al}$ ion in the center, while all but one (LU) lowest unoccupied orbitals are localized on the $n$-doped quantum dot with $\mathrm{P}$ ion in the center. In the bridged model, all highest occupied orbitals are localized on the $p$-doped quantum dot with $\mathrm{Al}$ ion in the center, while all lowest unoccupied orbitals are localized on the n-doped quantum dot with P ion in the center. In such configurations, the lowest energy excitations have charge transfer character.

Figure 6. Optical absorption spectra (x 50, filled area) and density of excited states (solid line) for eight atomic models of pairs of doped quantum dots, computed by TDDFT calculation with $\alpha_{\max }=200$ excited states for each model with the B3LYP functional and LANL2DZ basis. Absorption spectra tend to coincide with the density of excited states for bright excitations and vanish for charge transfer excitations. Panel labels are consistent with notation in Figure 3. Panels (a),(c),(e),(g) represent pairs of disconnected QDs. Panels (b),(d),(f),(h) represent bridged QDs chemically bonded to each other through bridging silicon atom. Panels (a)-(b) represent undoped QDs and labeled as intrinsic silicon (i-Si). Panels (c)-(d) represent aluminum doped QDs and are labeled as p-Si. Panels (e)-(f) represent phosphorus doped QDs and are labeled as n-Si. Panels (g)-(h) represent results for models where one QD in the pair is doped by aluminum and second one by phosphorous, therefore representing $p-n$ junction. Spectral signatures of donor $E_{d}$ and acceptor $E_{a}$ activation energies are indicated by arrows and are labeled. Sequence of peaks, their energies and heights establish qualitatively similar progressions in pairs of models (a)-(b), (c)-(d), (e)-(f), (h)-(g). Numerical values of $E_{g}$, $E_{a}, E_{d}, E_{c b-v b}$ are different in DFT and TDDFT calculations due to excitonic corrections.

Figure 7. TDDFT Transition energies and interpretation of four specific peaks in the density of excited states for disconnected and bridged models of $\mathrm{p}-\mathrm{n}$ junction. Based on comparison of single electron states and a density of excited states we interpret the four distinct peaks in Fig $\mathbf{6}(\mathrm{g})$ and $\mathbf{6}(\mathbf{h})$ as the following transitions ordered in the ascending of their energy: $\left(\mathbf{E}_{\mathbf{g}}\right)$ transitions between $\mathrm{p}$-state and n-state, providing lowest 
excitation at the bandgap and likely associated with larger charge transfer, $\left(\mathbf{E}_{\mathbf{g}}+\mathbf{E}_{\mathbf{d}}\right)$ an excitation where the hole is yet localized, while an electron is promoted higher up into the CB and becomes mobile. $\left(\mathbf{E}_{\mathbf{g}}+\mathbf{E}_{\mathbf{a}}\right)$ an excitation where the electron is yet localized, while a hole is promoted deeper down into the VB and becomes mobile, $\left(\mathbf{E}_{\mathbf{c b}}-\mathbf{E}_{\mathbf{v b}}\right)$ an excitation where both electron and hole are promoted into CB and VB and both become more mobile. Energies of all four peaks are consistently blueshifted by about $1 \mathrm{eV}$ in the bridged model. Negative Coulomb correction is smaller in bridged model, due to stronger screening.

Figure 8. Absolute value of lowest excitation transition densities projected on the axis connecting QDs. Panels (a)-(h) are labeled consistently with figures 3 and 6. Each panel represents the transition density for lowest excitation. Darkness of the spots is proportional to the absolute value of TD. The X-axis stands for position of electron, and the Y-axis represents the position of hole, according to Eqs. (6)-(9). (a) In disconnected, undoped dots, the lowest excitation is delocalized over both dots which witnesses forming a Förster excitonic band. (b) In connected, undoped dots, there is a substantial transition density on molecular junction atom. (c)-(f) The lowest excitation in each of single-type-doped model, $\mathrm{p}$ or $\mathrm{n}$, bridged or disconnected, has a degenerate charge transfer character. Amount of degeneracy is lower for (n-Si) bridged model. (according to Supplemental Fig. $\mathbf{S 4} \rightarrow \mathbf{S 3}$ ', higher excitations show VB-to-impurity character for n-doping and impurity-to-CB character for $\mathrm{p}$ doping). (g)-(h) p-n junction models, co-doped by $\mathrm{Al}$ and $\mathrm{P}$, disconnected and bridged, show similar features of transition density as follows: Nonzero transition density is present in lower right quadrant. The lowest excitation originates from aluminum (hole, lower half of the map) to phosphorous (electron, right half of the map). The lowest charge transfer excitation is represented by transition density mapped in the right lower quadrant of the transition density map. Both of the co-doped (p-n Si) models, disconnected and bridged, exhibit charge transfer excitons without degeneracy.

Figure 9.: Density of states of $\mathrm{Si}_{29}\left(\mathrm{CH}_{3}\right)_{36}$ QD calculated in (a) PBEPBE functional, (b) B3LYp and PBE1PBE and (c) CAM-B3LYP and LC-2PBE functionals. To show that the features are similar, breaks were applied in $\mathrm{x}$-axis in the graphs.

Figure 10. Absorption spectra of $\mathrm{Si}_{29}\left(\mathrm{CH}_{3}\right)_{36}$ QD using different functionals. (a) corresponds to PBE1PBE functional in vacuum and in acetonitrile solvent (b) corresponds to other functionals in vacuum as labelled. To align the bands, we shifted spectra by $-0.75 \mathrm{eV},+0.7 \mathrm{eV}$ and $-1.2 \mathrm{eV}$ for CAM-B3LYP, PBEPBE and LC$\omega \mathrm{PBE}$ functionalized calculations.

\section{$\underline{\text { IX. TABLES }}$}


Table 1.

\begin{tabular}{|c|c|c|c|c|c|c|c|c|}
\hline \multicolumn{2}{|r|}{ Model } & $\begin{array}{l}\text { Atomic } \\
\text { composition }\end{array}$ & $\mathrm{E}_{\mathrm{g}}$ & $\begin{array}{l}E_{c b}-E_{v b} \\
=E_{i}-E_{j}\end{array}$ & $\mathrm{i}$ & $\mathrm{j}$ & $\mathrm{E}_{\mathrm{d}}$ & $\mathrm{E}_{\mathrm{a}}$ \\
\hline \multirow{4}{*}{ 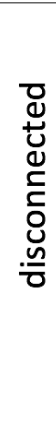 } & $i-S i$ & $2 \times \mathrm{Si}_{35} \mathrm{H}_{36}$ & 5.6654 & 5.6654 & LU & $\mathrm{HO}$ & N/A & N/A \\
\hline & $p-S i$ & $2 \times \mathrm{Al}_{1} \mathrm{Si}_{34} \mathrm{H}_{36}$ & .1760 & $\begin{array}{l}4.5551 / \\
5.2213^{*}\end{array}$ & $\mathrm{LU}+1$ & $\mathrm{HO}$ & N/A & .1760 \\
\hline & $n-S i$ & $2 \times \mathrm{P}_{1} \mathrm{Si}_{34} \mathrm{H}_{36}$ & .1744 & $\begin{array}{l}4.2174 / \\
4.9043^{*}\end{array}$ & LU & HO-1 & .1744 & N/A \\
\hline & $\begin{array}{l}p-n \\
\text { junction }\end{array}$ & $\begin{array}{l}\mathrm{Al}_{1} \mathrm{Si}_{34} \mathrm{H}_{36}+ \\
\mathrm{P}_{1} \mathrm{Si}_{34} \mathrm{H}_{36}\end{array}$ & 1.1951 & $\begin{array}{l}1.8971 / \\
2.6879^{* *}\end{array}$ & $\mathrm{LU}+1$ & HO-1 & $\begin{array}{l}.6835 \\
/ 1.8786^{* * *}\end{array}$ & $\begin{array}{l}.01850 / \\
1.2136^{* * * *}\end{array}$ \\
\hline \multirow{4}{*}{ 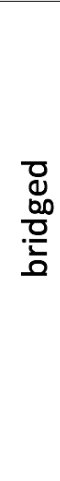 } & $i-S i$ & $\mathrm{H}_{34} \mathrm{Si}_{34}-\mathrm{Si}_{-} \mathrm{Si}_{34} \mathrm{H}_{34}$ & 5.3244 & 5.3244 & LU & $\mathrm{HO}$ & N/A & N/A \\
\hline & $p-S i$ & $\begin{array}{l}\mathrm{H}_{34} \mathrm{AlSi}_{34} \mathrm{Si}- \\
\mathrm{Si}_{34} \mathrm{AlH}_{34}\end{array}$ & .2851 & $\begin{array}{l}4.4332 / \\
5.0542^{*}\end{array}$ & $\mathrm{LU}+1$ & $\mathrm{HO}$ & N/A & .2851 \\
\hline & $n-S i$ & $\begin{array}{l}\mathrm{H}_{34} \mathrm{PSi}_{34}-\mathrm{Si}- \\
\mathrm{Si}_{34} \mathrm{PH}_{34}\end{array}$ & .8566 & $\begin{array}{l}2.6283 / \\
3.2882^{*}\end{array}$ & LU & HO- 1 & .8566 & N/A \\
\hline & $\begin{array}{l}p-n \\
\text { junction }\end{array}$ & $\begin{array}{l}\mathrm{H}_{34} \mathrm{AlSi}_{34}-\mathrm{Si}- \\
\mathrm{Si}_{34} \mathrm{PH}_{34}\end{array}$ & 2.2833 & $\begin{array}{l}2.9785 / \\
3.7369^{* *}\end{array}$ & LU+1 & HO-1 & .6710 & $\begin{array}{l}.02421 / \\
.78260\end{array}$ \\
\hline
\end{tabular}

Table 2.

\begin{tabular}{|l|l|l|l|l|}
\hline \multirow{2}{*}{$\begin{array}{l}\text { Number of bright } \\
\text { peak }\end{array}$} & \multicolumn{2}{|c|}{$\mathrm{p}-\mathrm{Si}$} & \multicolumn{2}{c|}{$\mathrm{n}-\mathrm{Si}$} \\
\cline { 2 - 5 } & $\begin{array}{l}\text { Fig. 6(c) } \\
2 \times \mathrm{Al}_{1} \mathrm{Si}_{34} \mathrm{H}_{36}\end{array}$ & $\begin{array}{l}\text { Literature* } \\
\mathrm{Al}_{1} \mathrm{Si}_{34} \mathrm{H}_{36}\end{array}$ & $\begin{array}{l}\text { Fig. 6(e) } \\
2 \times \mathrm{P}_{1} \mathrm{Si}_{34} \mathrm{H}_{36}\end{array}$ & $\begin{array}{l}\text { Literature** } \\
\mathrm{P}_{1} \mathrm{Si}_{34} \mathrm{H}_{36}\end{array}$ \\
\hline 1 & - & 0.4 & 0.9 & 1.0 \\
\hline 2 & $1.0,1.3$ & 1.0 & 1.7 & 1.7 \\
\hline 3 & 2.2 & 2.0 & 2.3 & 2.2 \\
\hline 4 & 2.6 & 2.7 & 2.7 & 2.7 \\
\hline 5 & $3.1,3.4$ & 3.3 & 3.5 & 3.2 \\
\hline 6 & 3.6 & 3.8 & 3.9 & 3.8 \\
\hline
\end{tabular}

Table 3. 


\begin{tabular}{|l|l|l|l|}
\hline $\begin{array}{l}\text { Number of } \\
\text { the peak }\end{array}$ & $\begin{array}{l}\text { Possible } \\
\text { interpretation }\end{array}$ & $\begin{array}{l}\text { Disconnected p-n- } \\
\text { junction } \\
\text { Fig. 6(g) }\end{array}$ & $\begin{array}{l}\text { Bridged p-n-junction } \\
\text { Fig. 6(h) }\end{array}$ \\
\hline 1 & $E_{g}, \mathrm{eV}$ & 1.0 & 2.1 \\
\hline 2 & $E_{g}+E_{d}, \mathrm{eV}$ & 1.7 & 2.7 \\
\hline 3 & $E_{g}+E_{a}, \mathrm{eV}$ & 2.0 & 3.0 \\
\hline 4 & $E_{c b-v b}, \mathrm{eV}$ & 2.6 & 3.6 \\
\hline
\end{tabular}

Table 4.

\begin{tabular}{|l|c|}
\hline \multicolumn{1}{|c|}{ Functional } & $\begin{array}{c}\text { Band Gap } \\
(\mathrm{eV})\end{array}$ \\
\hline B3LYP & 4.6243 \\
\hline CAM-B3LYP & 7.0352 \\
\hline PBEPBE & 3.3048 \\
\hline PBE1PBE & 4.8804 \\
\hline LC- $\omega$ PBE & 9.0140 \\
\hline
\end{tabular}

\section{$\underline{\text { X. FIGURES }}$}


Figure 1.

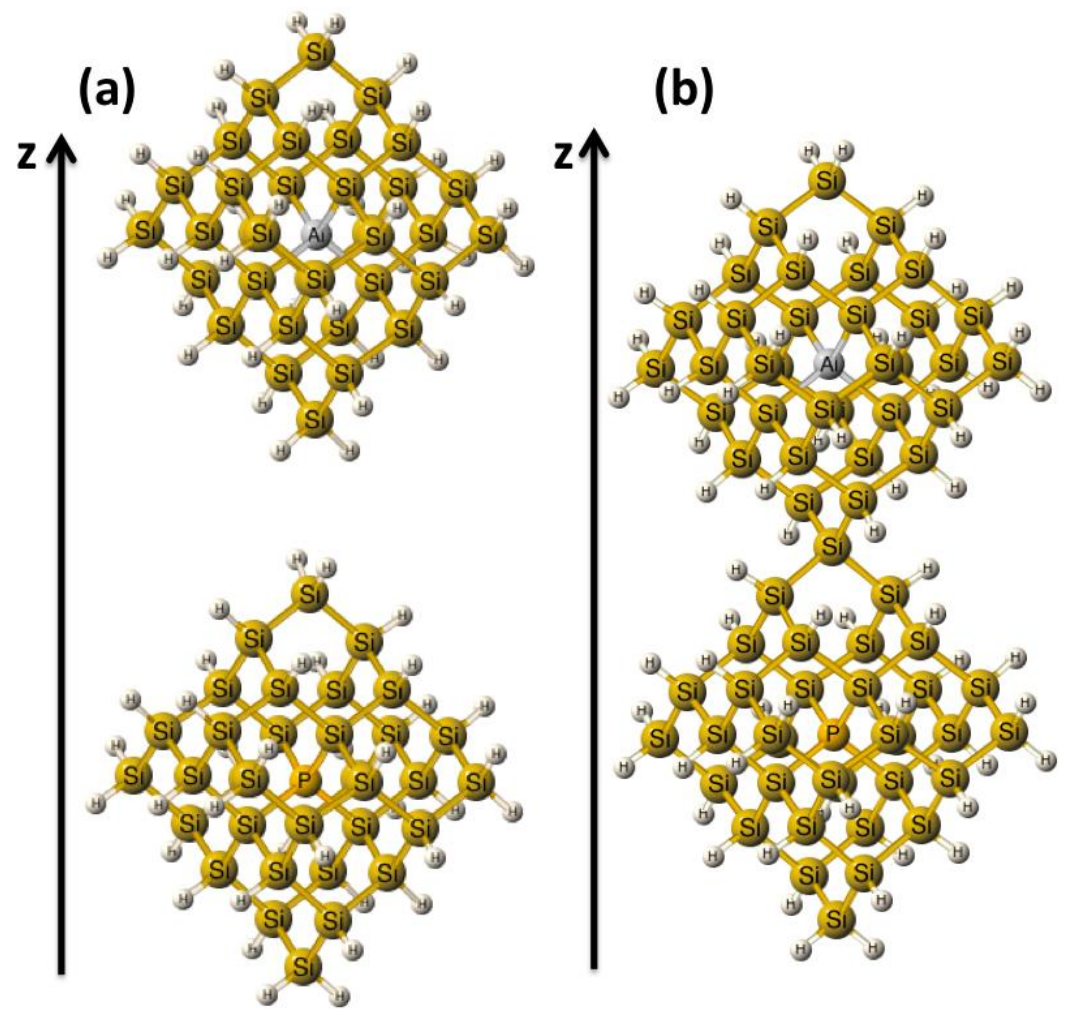

Figure 2. 


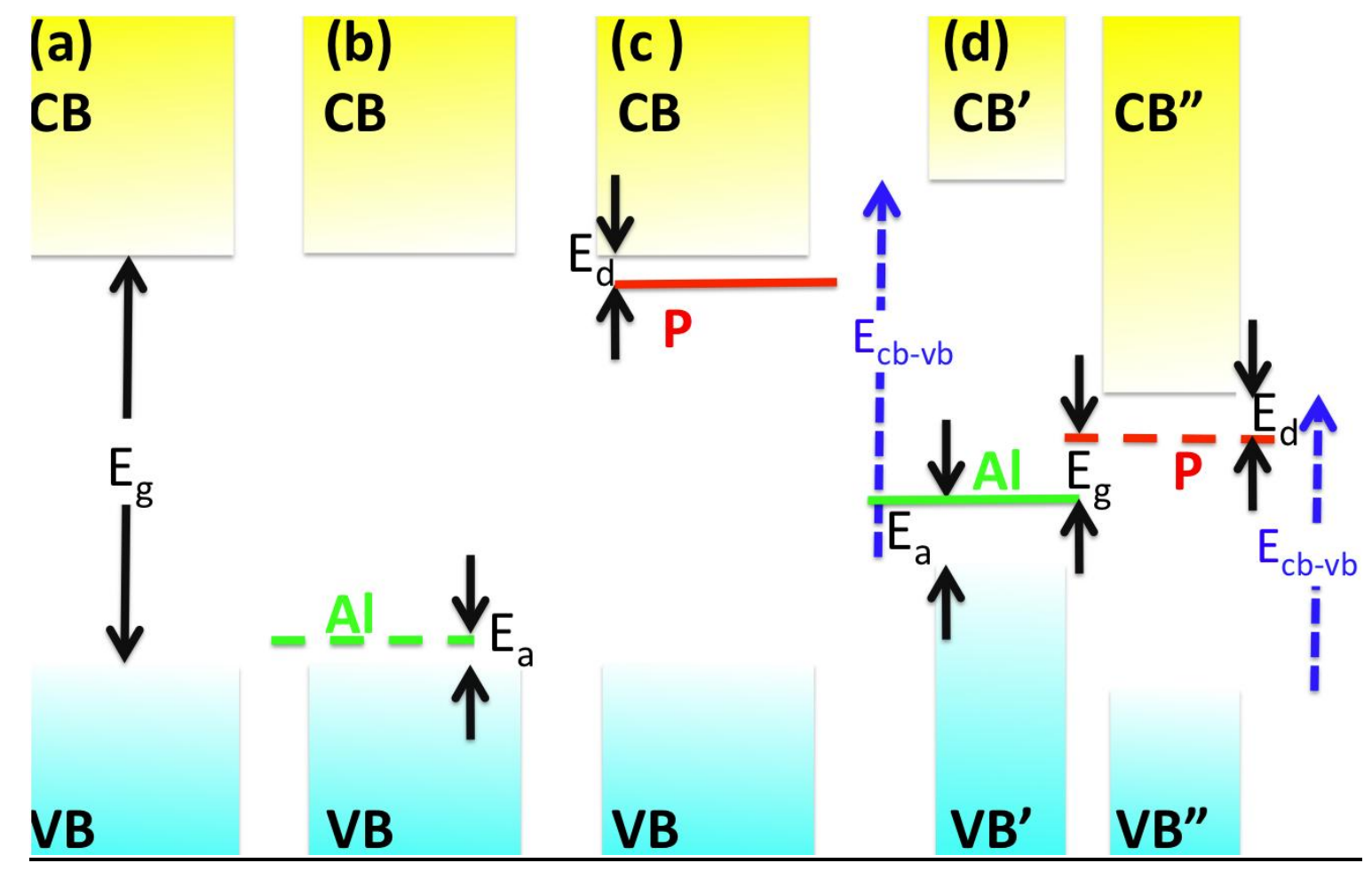

(e)

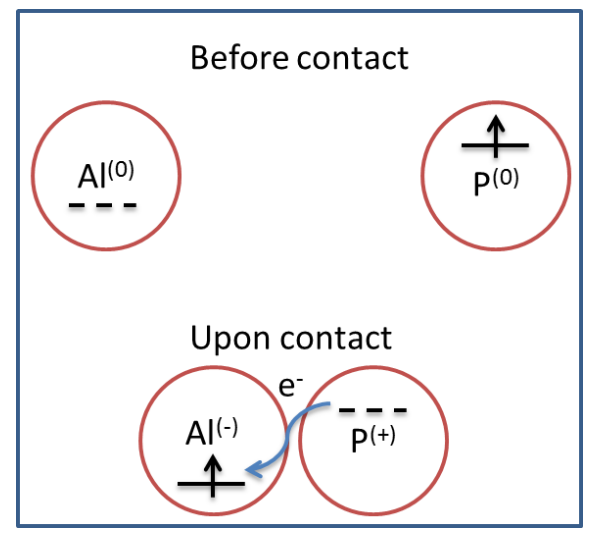

Figure 3. 


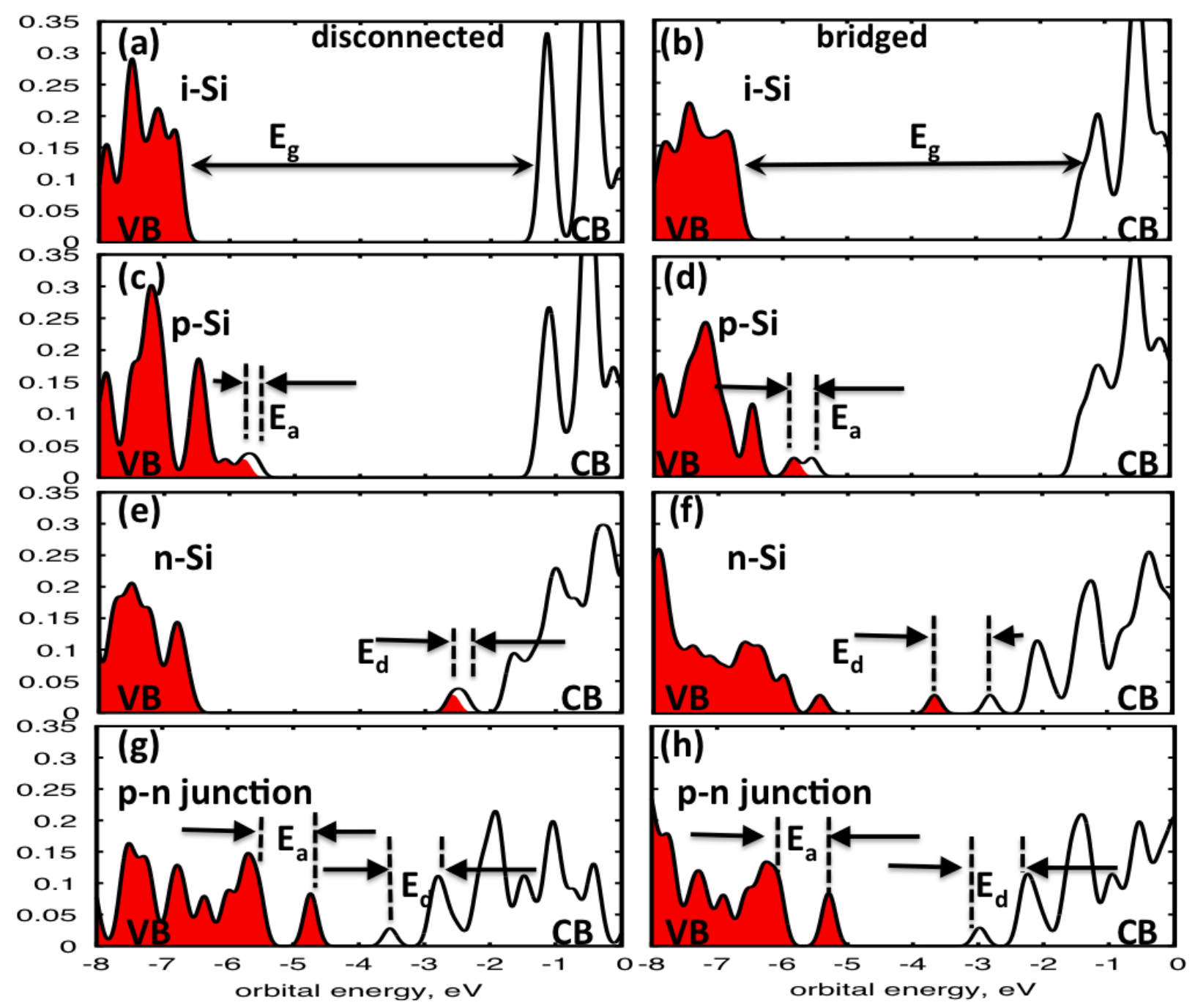

Figure 4.

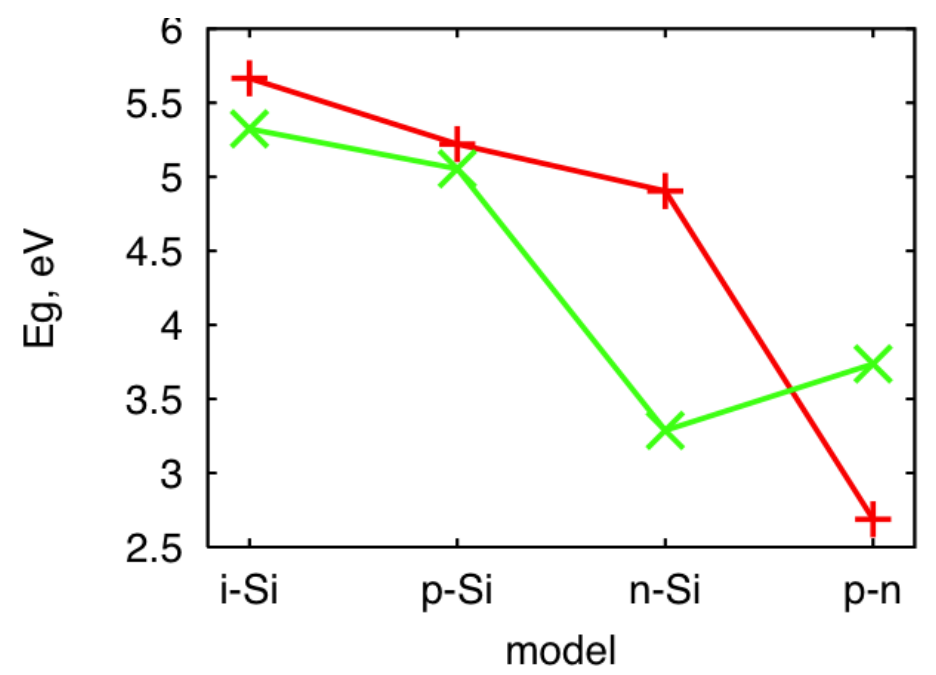

Figure 5. 


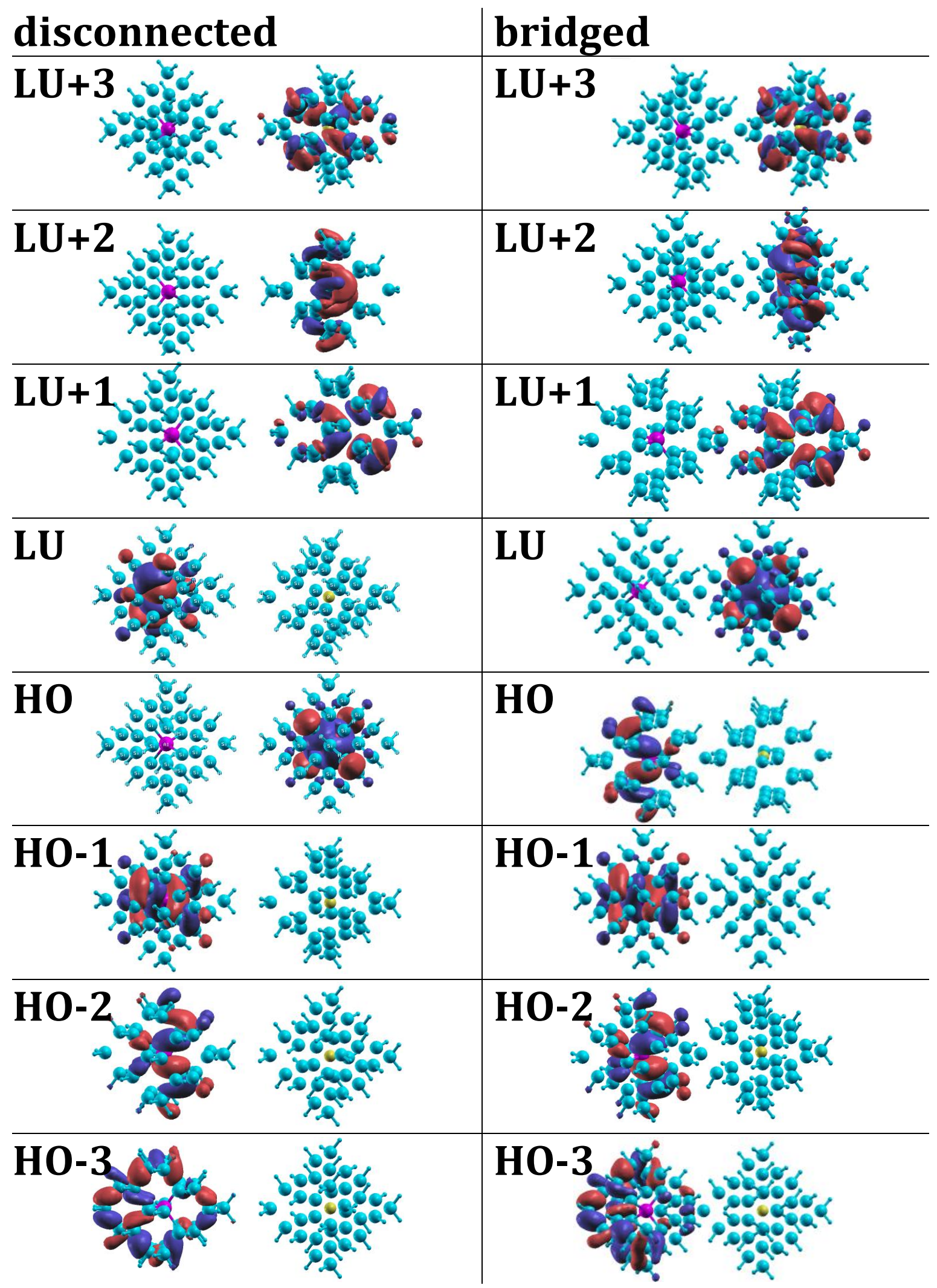

Figure 6. 

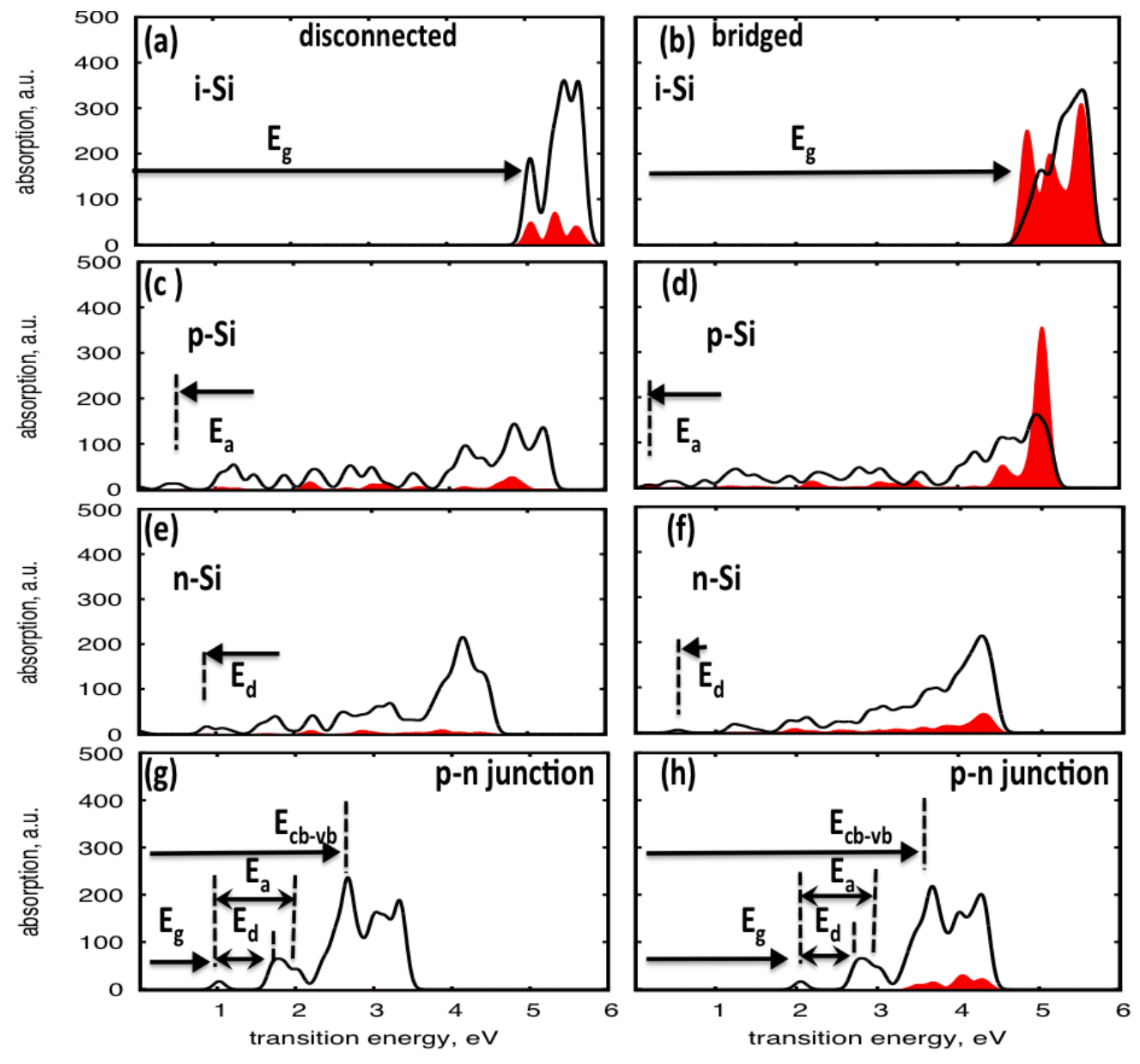

Figure 7.

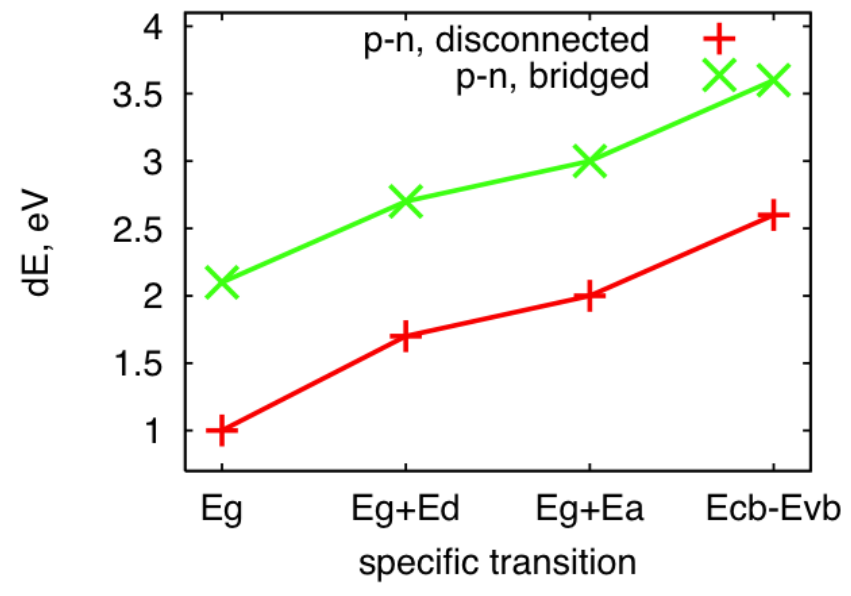


Figure 8 .
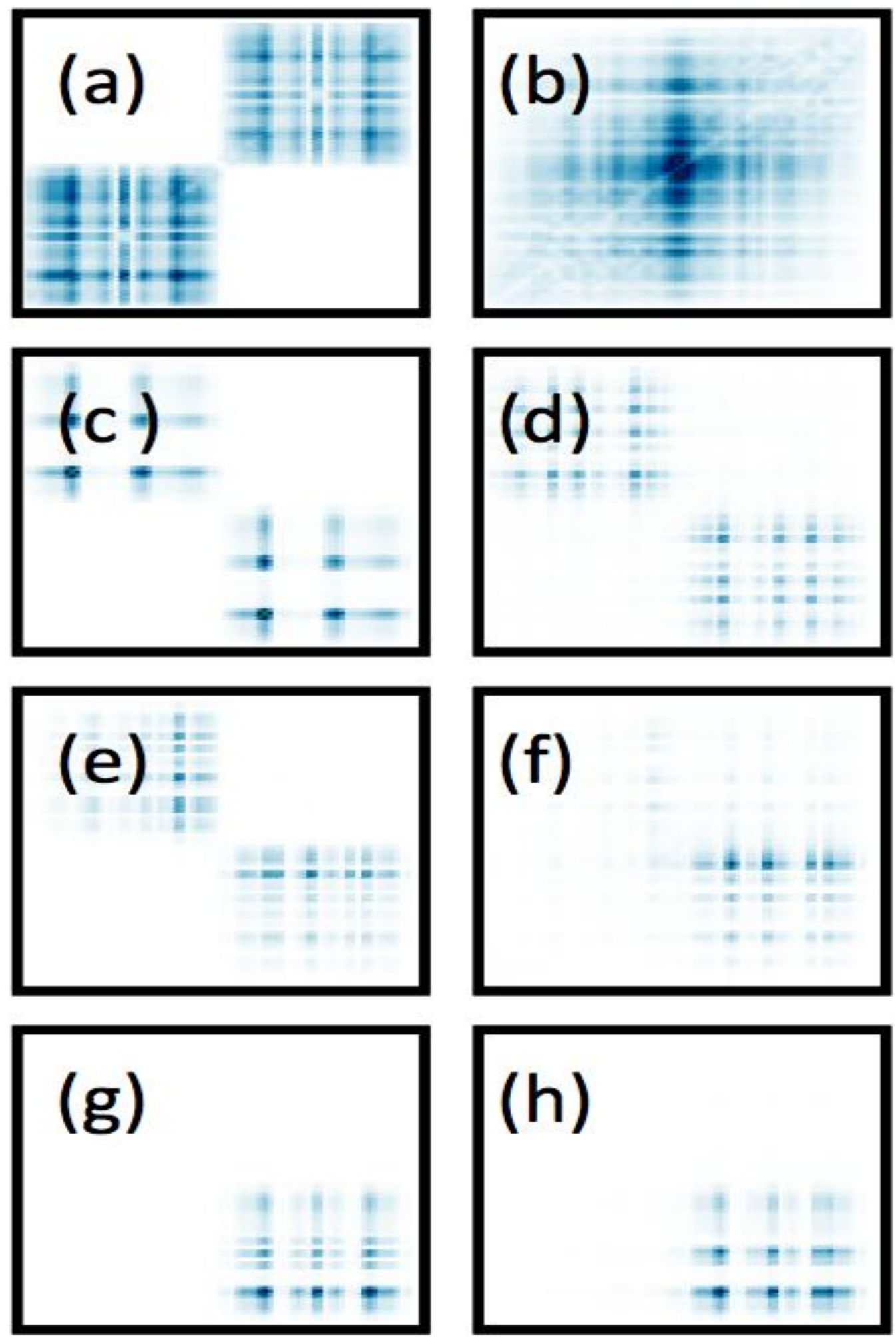
Figure 9.
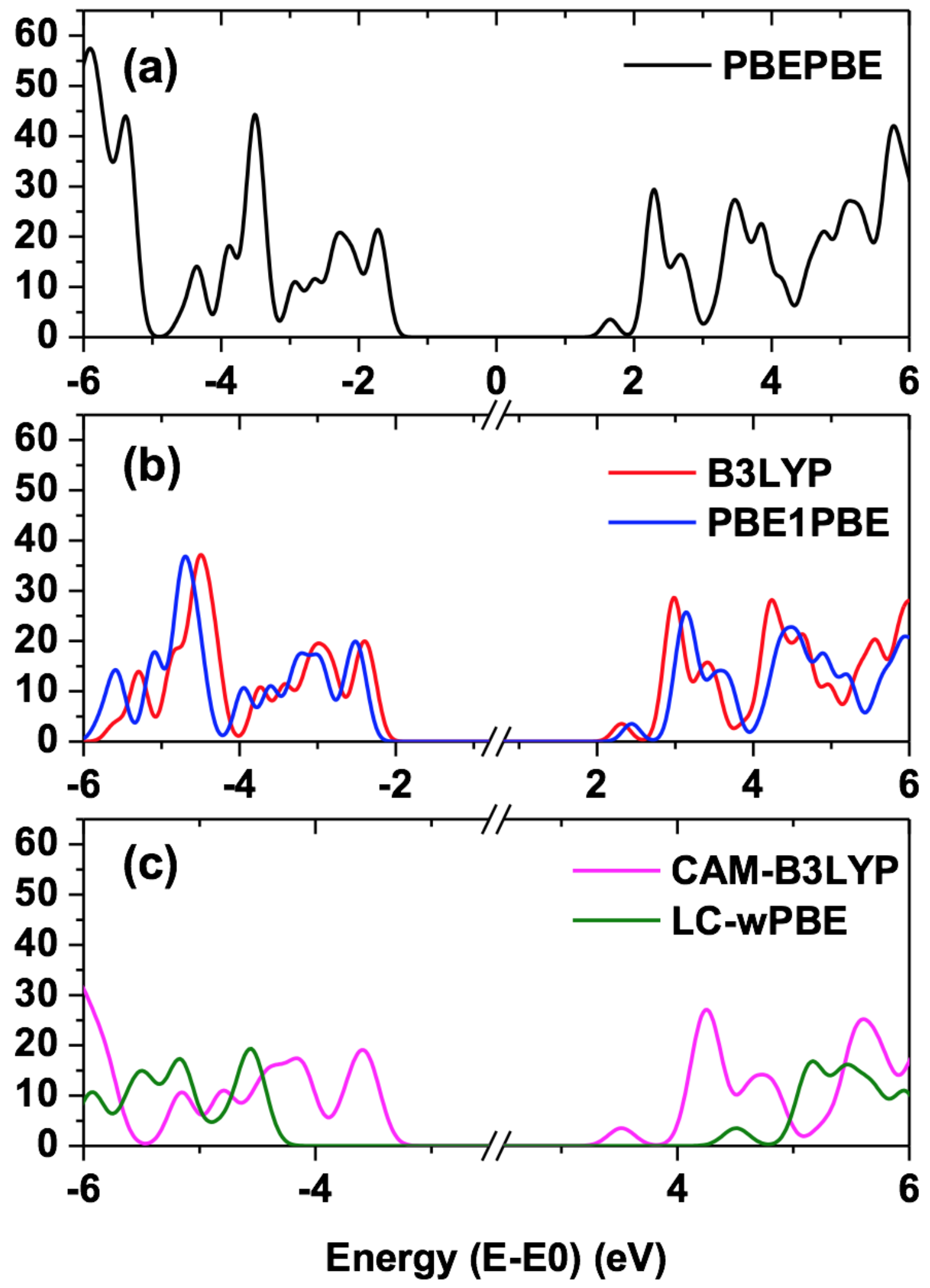
Figure 10.

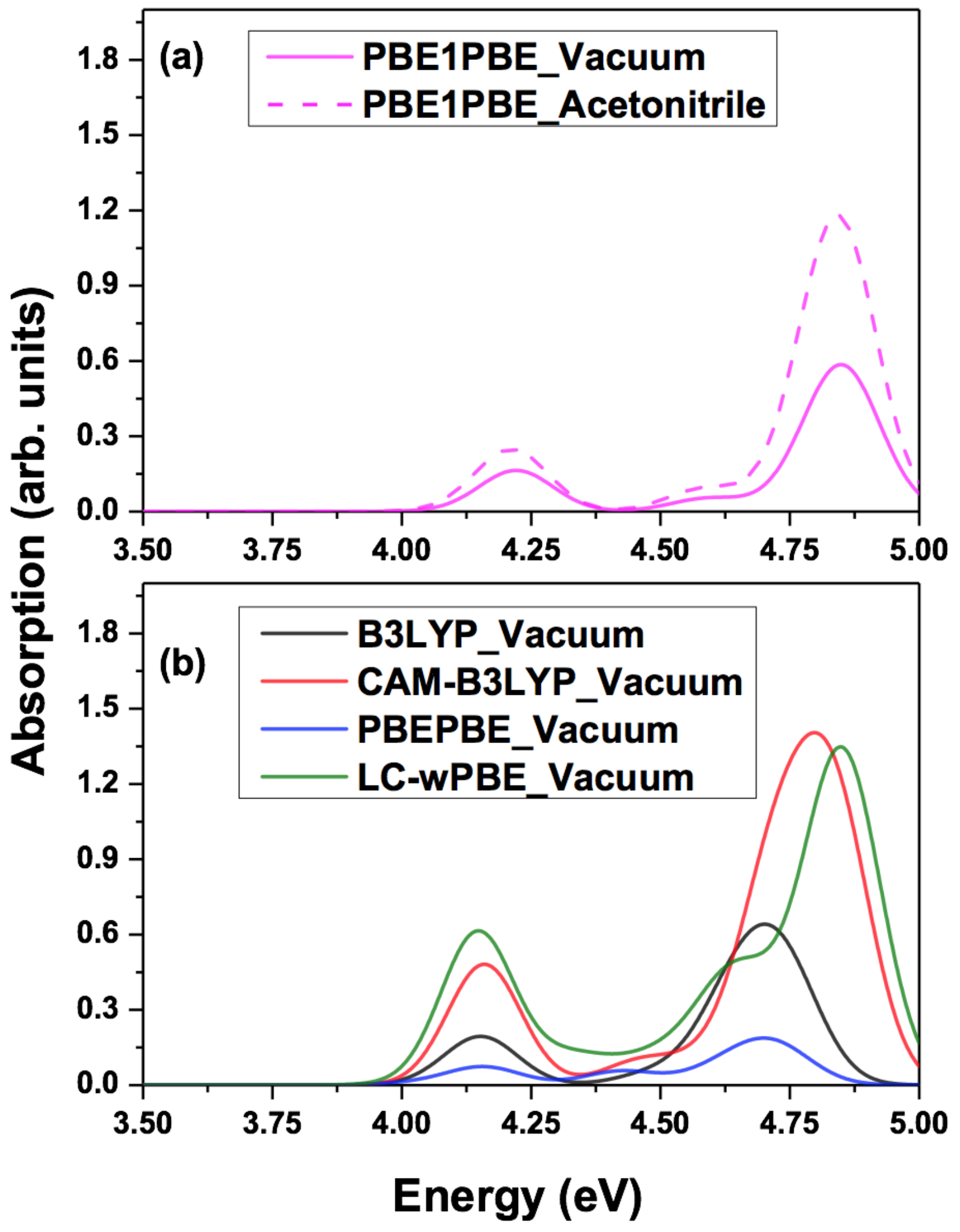




\section{XI.REFERENCES}

(1) Lu, Z. H.; Lockwood, D. J.; Baribeau, J. M. Nature 1995, 378, 258.

(2) Cheng, K. Y.; Anthony, R.; Kortshagen, U. R.; Holmes, R. J. Nano Lett. 2011, 11, 1952.

(3) Puzzo, D. P.; Henderson, E. J.; Helander, M. G.; Wang, Z. B.; Ozin, G. A.; Lu, Z. H. Nano Lett. 2011, 11, 1585.

(4) Brus, L. J. Phys. Chem. 1994, 98, 3575.

(5) Kohn, W. Solid State Physics-Advances in Research and Applications 1957, 5, 257.

(6) Shockley, W.; Queisser, H. J. J. Appl. Phys. 1961, 32, 510.

(7) Moore, G. E. Proceedings of the Ieee 1998, 86, 82.

(8) Huang, Y.; Duan, X. F.; Cui, Y.; Lauhon, L. J.; Kim, K. H.; Lieber, C. M. Science 2001, 294, 1313.

(9) Fuechsle, M.; Miwa, J. A.; Mahapatra, S.; Ryu, H.; Lee, S.; Warschkow, O.; Hollenberg, L. C. L.; Klimeck, G.; Simmons, M. Y. Nat. Nanotechnol. 2012, 7, 242.

(10) Prati, E.; Hori, M.; Guagliardo, F.; Ferrari, G.; Shinada, T. Nat. Nanotechnol. 2012, 7, 443.

(11) Janz, S.; Loper, P.; Schnabel, M. Materials Science and Engineering B-Advanced Functional Solid-State Materials 2013, 178, 542.

(12) Crooker, S. A.; Hollingsworth, J. A.; Tretiak, S.; Klimov, V. I. Phys. Rev. Lett. 2002, 89, 186802.

(13) Allan, G.; Delerue, C. Phys. Rev. B 2007, 75.

(14) Lin, Z.; Li, H.; Franceschetti, A.; Lusk, M. T. ACS Nano 2012, 6, 4029.

(15) Wolf, O.; Millo, O.; Balberg, I. J. Appl. Phys. 2013, 113.

(16) Kohn, W.; Luttinger, J. M. Physical Review 1955, 98, 915.

(17) Alberta, V. V.; Badaeva, E.; Kilina, S.; Sykora, M.; Tretiak, S. J. Lumin. 2011, 131, 1739.

(18) Proot, J. P.; Delerue, C.; Allan, G. Appl. Phys. Lett. 1992, 61, 1948.

(19) Takagahara, T.; Takeda, K. Phys. Rev. B 1992, 46, 15578.

(20) Wang, L. W.; Zunger, A. J. Phys. Chem. 1994, 98, 2158.

(21) Hill, N. A.; Whaley, K. B. Phys. Rev. Lett. 1995, 75, 1130.

(22) Delerue, C.; Lannoo, M.; Allan, G. Phys. Rev. Lett. 1996, 76, 3038.

(23) Trani, F.; Cantele, G.; Ninno, D.; Iadonisi, G. Phys. Rev. B 2005, 72.

(24) Raghavachari, K.; Rohlfing, C. M. J. Chem. Phys. 1991, 94, 3670.

(25) Fischer, S. A.; Prezhdo, O. V. Journal of Physical Chemistry C 2011, 115, 10006.

(26) Garoufalis, C. S.; Zdetsis, D.; Grimme, S. Phys. Rev. Lett. 2001, 87, 276402.

(27) Lehtonen, O.; Sundholm, D. J. Chem. Phys. 2006, 125.

(28) Puzder, A.; Williamson, A. J.; Grossman, J. C.; Galli, G. Phys. Rev. Lett. 2002, 88, 097401. 
(29) Williamson, A. J.; Grossman, J. C.; Hood, R. Q.; Puzder, A.; Galli, G. Phys. Rev. Lett. 2002, 89, 196803.

(30) Puzder, A.; Williamson, A. J.; Grossman, J. C.; Galli, G. J. Am. Chem. Soc. 2003, 125, 2786.

(31) Rohlfing, M.; Louie, S. G. Phys. Rev. Lett. 1998, 80, 3320.

(32) Noffsinger, J.; Kioupakis, E.; Van de Walle, C. G.; Louie, S. G.; Cohen, M. L. Phys. Rev. Lett. 2012, 108.

(33) Benedict, L. X.; Puzder, A.; Williamson, A. J.; Grossman, J. C.; Galli, G.; Klepeis, J. E.; Raty, J. Y.; Pankratov, O. Phys. Rev. B 2003, 68, 085310.

(34) Ogut, S.; Chelikowsky, J. R.; Louie, S. G. Phys. Rev. Lett. 1997, 79, 1770.

(35) Franceschetti, A.; Zunger, A. Phys. Rev. Lett. 1997, 78, 915.

(36) Wang, L. W.; Li, J. B. Phys. Rev. B 2004, 69, 153302.

(37) Casida, M. E.; Jamorski, C.; Bohr, F.; Guan, J. G.; Salahub, D. R. In Nonlinear Optical Materials: Theory and Modeling; Karna, S. P., Yeates, A. T., Eds.; Amer Chemical Soc: Washington, 1996; Vol. 628, p 145.

(38) Hirata, S.; Head-Gordon, M. Chem. Phys. Lett. 1999, 314, 291.

(39) Yanai, T.; Tew, D. P.; Handy, N. C. Chem. Phys. Lett. 2004, 393, 51.

(40) Heyd, J.; Scuseria, G. E.; Ernzerhof, M. J. Chem. Phys. 2003, 118, 8207.

(41) Vydrov, O. A.; Heyd, J.; Krukau, A. V.; Scuseria, G. E. J. Chem. Phys. 2006, 125.

(42) Grimme, S. J. Comput. Chem. 2006, 27, 1787.

(43) Mavros, M. G.; Micha, D. A.; Kilin, D. S. Journal of Physical Chemistry C 2011, 115, 19529.

(44) Bruno, M.; Palummo, M.; Ossicini, S.; Del Sole, R. Surf. Sci. 2007, 601, 2707.

(45) Rurali, R. Reviews of Modern Physics 2010, 82, 427.

(46) Fernandez-Serra, M. V.; Adessi, C.; Blase, X. Nano Lett. 2006, 6, 2674.

(47) Fernandez-Serra, M. V.; Adessi, C.; Blase, X. Phys. Rev. Lett. 2006, 96.

(48) Niquet, Y. M.; Lherbier, A.; Quang, N. H.; Fernandez-Serra, M. V.; Blase, X.; Delerue, C. Phys. Rev. B 2006, 73.

(49) Kryjevski, A.; Kilin, D.; Kilina, S. Journal of Renewable Sustainable Energy 2013, 5 043120.

(50) Kilin, D. S.; Micha, D. A. Chem. Phys. Lett. 2008, 461, 266.

(51) Kilin, D. S.; Micha, D. A. Journal of Physical Chemistry C 2009, 113, 3530.

(52) Vazhappilly, T.; Kilin, D. S.; Micha, D. A. Journal of Physical Chemistry C 2012, 116, 25525 .

(53) Chen, J. C.; Schmitz, A.; Kilin, D. S. Int. J. Quantum Chem 2012, 112, 3879.

(54) Chen, J.; Schmitz, A.; Inerbaev, T.; Meng, Q.; Kilina, S.; Tretiak, S.; Kilin, D. S. Journal of Physical Chemistry Letters 2013, 42906. 
Zhang, R. Q.; De Sarkar, A.; Niehaus, T. A.; Frauenheim, T. Physica Status Solidi B-Basic Solid State Physics 2012, 249, 401.

(56) Guerra, R.; Ossicini, S. Phys. Rev. B 2013, 87, 165441.

(57) Govoni, M.; Marri, I.; Ossicini, S. Nature Photonics 2012, 6, 672.

(58) Gali, A.; Voros, M.; Rocca, D.; Zimanyi, G. T.; Galli, G. Nano Lett. 2009, 9, 3780.

(59) Seino, K.; Bechstedt, F.; Kroll, P. Phys. Rev. B 2012, 86.

(60) Beard, M. C.; Knutsen, K. P.; Yu, P.; Luther, J. M.; Song, Q.; Metzger, W. K.; Ellingson, R. J.; Nozik, A. J. Nano Lett. 2007, 7, 2506.

(61) Timmerman, D.; Izeddin, I.; Stallinga, P.; Yassievich, I. N.; Gregorkiewicz, T. Nature Photonics 2008, 2, 105.

(62) Timmerman, D.; Valenta, J.; Dohnalova, K.; de Boer, W. D. A. M.; Gregorkiewicz, T. Nat. Nanotechnol. 2011, 6, 710.

(63) Forster, T. Annalen Der Physik 1948, 2, 55.

(64) Dexter, D. L. J. Chem. Phys. 1953, 21, 836.

(65) Baer, R.; Rabani, E. J. Chem. Phys. 2008, 128.

(66) Jain, P. K.; Ghosh, D.; Baer, R.; Rabani, E.; Alivisatos, A. P. Proceedings of the National Academy of Sciences of the United States of America 2012, 109, 8016.

(67) Frisch, M. J.; Trucks, G. W.; Schlegel, H. B.; Scuseria, G. E.; Robb, M. A.; Cheeseman, J. R.; Scalmani, G.; Barone, V.; Mennucci, B.; Petersson, G. A.; Nakatsuji, H.; Caricato, M.; Li, X. H., H. P.; Izmaylov, A. F.; Bloino, J.; Zheng, G.; Sonnenberg, J. L.; Hada, M.; Ehara, M.; Toyota, K.; Fukuda, R.; Hasegawa, J.; Ishida, M.; Nakajima, T.; Honda, Y.; Kitao, O.; Nakai, H.; Vreven, T.; Montgomery Jr., J. A.; Peralta, J. E.; Ogliaro, F.; Bearpark, M.; Heyd, J. J.; Brothers, E.; Kudin, K. N.; Staroverov, V. N.; Kobayashi, R.; Normand, J.; Raghavachari, K.; Rendell, A.; Burant, J. C.; Iyengar, S. S.; Tomasi, J.; Cossi, M.; Rega, N.; Millam, N. J.; Klene, M.; Knox, J. E.; Cross, J. B.; Bakken, V.; Adamo, C.; Jaramillo, J.; Gomperts, R.; Stratmann, R. E.; Yazyev, O.; Austin, A. J.; Cammi, R.; Pomelli, C.; Ochterski, J. W.; Martin, R. L.; Morokuma, K.; Zakrzewski, V. G.; Voth, G. A.; Salvador, P.; Dannenberg, J. J.; Dapprich, S.; Daniels, A. D.; Farkas, Ö.; Foresman, J. B.; Ortiz, J. V.; Cioslowski, J.; Fox, D. J.; Revision A.2 ed.; Gaussian, Inc.: Wallingford, CT, 2009.

(68) Tretiak, S.; Mukamel, S. Chem. Rev. 2002, 102, 3171.

(69) Mukamel, S.; Tretiak, S.; Wagersreiter, T.; Chernyak, V. Science 1997, 277, 781.

(70) Tretiak, S.; Zhang, W. M.; Chernyak, V.; Mukamel, S. Proceedings of the National Academy of Sciences of the United States of America 1999, 96, 13003.

(71) Wang, S. J.; Bazan, G. C.; Tretiak, S.; Mukamel, S. J. Am. Chem. Soc. 2000, 122, 1289.

(72) Tretiak, S.; Kilina, S.; Piryatinski, A.; Saxena, A.; Martin, R. L.; Bishop, A. R. Nano Lett. 2007, 7, 86.

(73) Kilina, S.; Tretiak, S. Adv. Funct. Mater. 2007, 17, 3405. 
(74) Brown, S. L.; Vogel, D. J.; Miller, J. B.; Inerbaev, T. M.; Anthony, R. J.; Kortshagen, U. R.; Kilin, D. S.; Hobbie, E. K. The Journal of Physical Chemistry C 2016.

(75) Shockley, W. The Bell System Technical Journal 1949, 28, 435.

(76) Ramdas, A. K.; Rodriguez, S. Rep. Prog. Phys. 1981, 44, 1297.

(77) Zhou, Z. Y.; Steigerwald, M. L.; Friesner, R. A.; Brus, L.; Hybertsen, M. S. Phys. Rev. B

2005, 71 .

(78) Iori, F.; Degoli, E.; Magri, R.; Marri, I.; Cantele, G.; Ninno, D.; Trani, F.; Pulci, O.; Ossicini, S. Phys. Rev. B 2007, 76.

(79) Ossicini, S.; Degoli, E.; Iori, F.; Luppi, E.; Magri, R.; Cantele, G.; Trani, F.; Ninno, D. Appl Phys Lett 2005, 87.

(80) Cantele, G.; Degoli, E.; Luppi, E.; Magri, R.; Ninno, D.; Iadonisi, G.; Ossicini, S. Phys. Rev. $B \mathbf{2 0 0 5}, 72$.

(81) Fischer, S. A.; Crotty, A. M.; Kilina, S. V.; Ivanov, S. A.; Tretiak, S. Nanoscale 2012, 4, 904.

(82) Kilina, S.; Ivanov, S.; Tretiak, S. J. Am. Chem. Soc. 2009, 131, 7717.

(83) Kilina, S.; Kilin, D.; Tretiak, S. Chem. Rev. 2015, 115, 5929. 